\title{
Extended optical spectroscopic monitoring of wind structure in HD 152408*
}

\author{
R. K. Prinja ${ }^{1}$, O. Stahl ${ }^{2}$, A. Kaufer ${ }^{3}$, S. R. Colley ${ }^{1}$, P. A. Crowther ${ }^{1}$, and B. Wolf ${ }^{2}$ \\ 1 Department of Physics \& Astronomy, University College London, Gower Street, London WC1E 6BT, UK \\ 2 Landessternwarte Heidelberg, Königstuhl 12, 69117 Heidelberg, Germany \\ 3 European Southern Observatory, Alonso de Cordova 3107, Santiago 19, Chile
}

Received 25 August 2000 / Accepted 14 December 2000

\begin{abstract}
New perspectives are provided on significant spatial structure and temporal variability in the nearstar wind regions (i.e. $<3 R_{\star}$ ) of the massive luminous star HD 152408 (classified as O8:Iafpe or WN9ha). This study is primarily based on the analysis of high-quality échelle spectra secured during 21 nights between 1999 July to August, using the Landessternwarte-developed (fibre-fed) FEROS instrument on the ESO 1.52-m telescope. These extended time-series data, with a total simultaneous wavelength coverage of $\lambda \lambda 3600-9200 \AA$, were exploited to monitor absorption and emission fluctuations (of $\sim 5-10 \%$ of the line flux) in several He I and Balmer lines, together with more deep-seated (near-photosphere) disturbances in weaker metallic emission and absorption lines. Organised large-scale wind structure in HD 152408 is principally betrayed by sequential episodes of discrete absorption and emission features, which migrate from near zero velocity to almost the wind terminal velocity. This evolution is extremely slow, however, typically spanning $\sim 4$ days for an individual episode. We demonstrate that the blue-shifted absorption episodes in He I are very closely mirrored (in velocity and time) by absorption features (i.e. reduced not enhanced flux) in the blue wings of the mainly recombination formed broad $\mathrm{H} \alpha$ emission line. The implication is that there is detailed balancing between ground state photoionization and recombination in the substantially optically thick Balmer lines. Surprisingly, the velocity behaviour of the red-ward and blue-ward migrating features is highly asymmetric, such that the mean acceleration of the former is less than $50 \%$ of the latter. Fourier analysis reveals a modulation time-scale for the wind activity of $\sim 7.7$ days, plus its harmonic at 3.9 days. The longer period is $\sim 28$ times greater than the characteristic radial wind flow time of HD 152408. We also detect a $\sim 1.5$ day periodic variation in the radial velocity of the weak C IV $\lambda \lambda 5801,5812$ absorption lines, which are the closest approximation to "pure" photospheric lines in the optical spectrum of HD 152408. The wind-formed optical lines of HD 152408 are also affected by fluctuations in the central peak emission, particularly evident in $\mathrm{H} \alpha$ where the equivalent width may vary by up to $20 \%$. Data secured between 1995 and 1999 reveal, however, that the epoch-to-epoch mean profiles are remarkably similar. Non-LTE steadystate stellar atmosphere models are used to synthesis profiles to match representative $\mathrm{H} \alpha$ and $\mathrm{He}$ I $\lambda 5876$ line profiles. Only a slow (tailored) velocity law (compared to $\beta=1$ ) provides a good match to the $\mathrm{H} \alpha$ emission peak and wings, but the models predict excess He I absorption. The observed extreme $\mathrm{H} \alpha$ emission variations can be reproduced by the synthetic profiles with an implied $\pm 10 \%$ variation in mass-loss rate. The results on optical line profile variability in HD 152408 are discussed in the context of models for co-rotating interaction regions (CIRs) in the wind. Several constraints are provided that argue against simple velocity fields in such streams, including (i) the slow acceleration of features to high velocities, within $\sim 3 R_{\star}$, (ii) the strong asymmetry in projected acceleration of the approaching and receding stream material, (iii) Balmer line absorption effects in the approaching material, (iv) the rise of localised features from very low velocities, and (v) the stability of the large-scale CIRs against turbulent small-scale wind structure. We suggest that it may be worth exploring hydrodynamic simulations of CIRs that incorporate different velocity fields on the leading (faster accelerating; blue-ward absorption) and trailing (slower accelerating; red-ward emission) edges of the spiral structures.
\end{abstract}

Key words. line: profiles - stars: early-type - stars: individual (HD 152408) - stars: mass loss

\section{Introduction}

Send offprint requests to: R. K. Prinja,

e-mail: rkp@star.ucl.ac.uk

* Based on observations collected at the European Southern Observatory, Chile (ESO 63.H-0080(A)).
Time-series optical and ultraviolet (UV) spectroscopy has unambiguously established that the radiation-pressuredriven winds of luminous early-type (OB) stars are highly 
variable on characteristic time-scales of hours to several days. The most substantial fluctuations seen in the absorption and emission line profiles of these stars betray organised large-scale structure in the winds, which may be induced by changes in the star itself and can have an impact on the overall nature of the outflow. The principal tracers of large-scale wind perturbations in OB star winds are localised evolving features that provide optical depth and velocity structure to otherwise smooth windformed spectral lines. These diagnostics have been most sensitively exploited via time-series monitoring of UV resonance lines with the International Ultraviolet Explorer $(I U E)$ satellite. The latest (and final) of these campaigns used IUE to study the winds of a few Galactic OB stars almost continuously for several weeks (e.g. Massa et al. 1995; Kaper et al. 1996; Prinja et al. 1998). The extended monitoring revealed that the wind line profiles vary continuously and provided striking evidence for repeatability in wind activity over a typical time-scale of several days. Generally, the modulation times for the wind variability are substantially longer than the wind flow times ( $\sim$ hours). These results have led to the notion that largescale wind structure in OB stars may be directly linked to the stellar rotation rate, with a causal connection to inhomogeneities at the stellar surface.

A currently popular model for providing slowly propagating and repetitive wind perturbations in hot stars is based on earlier discussions by Mullan $(1984,1986)$ of the potential role of co-rotating interaction regions (CIRs) in massive stars. Cranmer \& Owocki (1996) developed these ideas in detail for the case of OB stars and consider inhomogeneities which cause the wind from different longitudinal sectors on the stellar surface to emerge with different densities and/or velocities. These different wind streams then interact to form CIRs, which inevitably evolve into spiral-shaped shock structures. In this model, variations in wind absorption result from material flowing through a semi-permanent co-rotating wind structure.

The applicability of the CIR model is not widely tested, however, and challenging issues remain concerning the physical coupling of inhomogeneities at the stellar surface to the generation of wind structure. The impact of substantial wind variability on mass-loss rate estimates also needs further exploration. A particular problem is that though the $I U E$ satellite produced the landmark data sets mentioned above, the termination of its operations in 1996 left the results on hot star wind structure on a fairly narrow astrophysical footing. Ultraviolet data sets of requisite high spectral and temporal resolution, extending over 15 days or more, only exist for about half-a-dozen $\mathrm{O}$ and early B supergiants. The detailed time-dependent spectroscopic properties can differ substantially, however, from star to star. For example, the nature of the features responsible for providing modulated (or even cyclic) wind activity can differ fundamentally between "classical" discrete absorption components (DACs, e.g. $\xi$ Per, De Jong 2000), near-sinusoidal localised variations about a well defined mean state (HD 64760, Fullerton et al. 1997), and fluctuations of the entire Doppler shifted absorption trough ( $\zeta$ Pup, Howarth et al. 1995). Furthermore, one of the observational signatures relied on in the CIR-type models is "phase-bowing" in the UV wind lines, where discrete features in the line profiles develop simultaneously towards lower and higher blue-shifted velocities. (see e.g. Fullerton et al. 1997). This property is, however, a function of the ratio of rotation velocity to wind terminal velocity, and is absent in most of the other stars studied.

Proper understanding of the outflows of OB stars requires a deeper knowledge of the nature and consequences of their variability. This is also important since the origin of large-scale wind structure has implications for the fundamental nature of the stellar surfaces. In the post- $I U E$ era, key observational diagnostics can still be obtained at several wave-bands, including optical, infrared, millimetre and radio. In fact complementary data from these different regions are extremely important to determine the form of wind structure on different geometric scales. Nevertheless, optical spectroscopy currently offers the more direct diagnostics of large-scale wind perturbations in OB stars (see e.g. Ebbets 1982; Kaper et al. 1997, 1998). In particular, the density-squared dependence of the recombination lines makes them ideal probes of the inner-wind regions (less than $3 R_{\star}$ ) and have the potential to tightly constrain the depth (close to the photosphere) to which large-scale structure persists in the winds. With this motivation, we present in this paper échelle optical time-series data of the massive luminous star HD 152408, spanning over 20 nights in 1999, plus more limited data taken several years apart. Fresh perspectives are provided on hot star wind structure by studying deep-seated activity simultaneously in a variety of strategic lines, including the Balmer series, He I, He II, and near-photospheric (relatively weak) metal absorption and emission lines. Our study differs fundamentally from recent optical spectroscopic work on smallscale wind structure, which is usually identified as rapidly evolving (over $\sim$ hours) bumps on "pure" emission profiles (e.g. Moffat \& Robert 1992; Eversberg et al. 1998). We identify in particular slowly migrating (over $\sim$ several days) absorption signatures due to large-scale features; the temporal behaviour of wind material localised along the line of sight to the stellar disk is not only examined in He absorption troughs, but it can also be seen acting on the blue $\mathrm{H} \alpha$ "emission" wing.

\subsection{The candidate star, $H D 152408$}

HD 152408 is a luminous member of NGC 6231, and is believed to be a single star (e.g. Conti et al. 1977; $m_{V} \sim 5.8$ ). It was originally classified as O8:Iafpe by Walborn (1972), but this has been revised to WN9ha by Bohannan \& Crowther (1999; see also Walborn \& Fitzpatrick 2000). A detailed atmospheric analysis of the optical spectrum was also presented by Crowther \& Bohannan (1997), who support the general notion that Of stars are helium enriched by core processed material. HD 152408 has a strong 
Table 1. Stellar parameters of HD 152408

\begin{tabular}{lcc}
\hline Parameter & Value & Reference \\
\hline Spectral type & O8:Iafpe, WN9ha & 1,6 \\
$T_{\text {eff }}$ & $28500 \mathrm{~K}$ & 2 \\
$\log \left(L / L_{\odot}\right)$ & 5.80 & 2 \\
$\dot{M}($ mean $)$ & $2.110^{-5} M_{\odot} / \mathrm{yr}$ & 3 \\
$v_{\infty}$ & $955 \mathrm{~km} \mathrm{~s}^{-1}$ & 4 \\
$v_{\mathrm{e}} \sin (\mathrm{i})$ & $85 \mathrm{~km} \mathrm{~s}^{-1}$ & 5 \\
$P_{\text {rot }} / \sin (\mathrm{i})$ & 20 days & - \\
\hline
\end{tabular}

1: Walborn (1972); 2: Crowther \& Bohannan (1997); 3: this paper; 4: Prinja et al. (1990); 5: Howarth et al. (1997); 6: Bohannan \& Crowther (1999)

stellar wind (e.g. Swings 1942; Struve 1944; Walborn 1975; Fullerton 1990), and the star provides an interesting opportunity to "bridge" the wind properties of very luminous O-type stars and late WN-type Wolf-Rayet stars. HD 152408 offers rich prospects in the optical for comparisons between blue-shifted absorption and extended emission line profiles, which we exploit here in our study of its temporal wind properties. Previously, Prinja \& Fullerton (1994) reported on a short ( $\sim 5$ night) optical time-series data set of this star, highlighting blue-ward migrating discrete absorption features in the trough of He I $\lambda 5876$, with similar characteristics to slowly evolving DACs seen in UV spectra of OB stars. Additional evidence for deep atmospheric or photospheric activity is signalled by the photometric variations reported by Balona (1982; see also Morrison 1975), which range up to $\sim 0.02 \mathrm{mag}$ in $V$ over several nights; there is no clear evidence in these data for periodic modulations. A summary of stellar parameters is given in Table 1. Note that HD 152408 has an exceptionally low ratio of $v_{\infty} / v_{\text {edge }} \sim 0.43$ (where $v_{\text {edge }}$ is the velocity at which the gradually sloped blue wings of the UV resonance lines meet the local continuum). This value is discrepant with that for Galactic O stars generally, and is only barely matched by a few WR stars (e.g. Prinja et al. 1990).

\section{The optical data sets}

Our study of time-dependent wind activity in HD 152408 is principally based on observations secured between 1999 July 13 and 1999 August 4 using the Fibre-fed Extended Range Optical Spectrograph (FEROS), which was developed and built for the European Southern Observatory (ESO) by a consortium led by the Landessternwarte Heidelberg (Kaufer et al. 2000). Our data set was secured during Guaranteed Time observations, with FEROS mounted on the ESO $1.52 \mathrm{~m}$ telescope at La Silla, Chile. The spectrograph provides simultaneous coverage over a substantial range from 3600 to $9200 \AA$ in 39 orders, with a resolving power, $\lambda / \Delta \lambda=48000$ (2.2. pixels). A $2 \mathrm{~K} \times 4 \mathrm{~K}$ (15 $\mu$ pixel) EEV CCD detector was used. The individual observations of HD 152408 were secured (by A.K. and
Table 2. Summary of the optical data sets

\begin{tabular}{llc}
\hline Instrument & \multicolumn{1}{c}{ Dates } & No. of spectra \\
\hline FEROS & 1999 July 13-Aug. 4 & 81 \\
HEROS & 1995 May 20-June 3 & 15 \\
HEROS & 1996 April 18-June 1 & 15 \\
HEROS & 1999 July 4-July 10 & 7 \\
\hline
\end{tabular}

O.S.) with typical exposure times of $\sim 10 \mathrm{~min}$, and signalto-noise in the range $\sim 175-200$ (at $\lambda \lambda 6630$ to $6650 \AA$ ).

The time-series analysis presented here is based on 81 FEROS spectra taken over 21.25 days. The sampling was typically 4 to 5 spectra taken over $\sim 7$ hours each night, with the exception of a data gap of 2.76 days between 1999 July 17 and July 20. FEROS was delivered at ESO with pipe-line data reduction software (e.g. Kaufer et al. 2000), and these ESO-MIDAS based algorithms ("context feros") were used to process the spectra according to standard échelle reduction procedures, including bias subtraction, flat fielding, correction for scattered light and order overlaps, wavelength calibration and optimal extraction of the spectra. Heliocentric velocities are presented throughout this paper; we opt against transforming to an approximate stellar rest frame by subtracting a radial velocity of $\sim-40 \mathrm{~km} \mathrm{~s}^{-1}$ (e.g. Conti et al. 1977; Fullerton 1990), since we find evidence here for systematic radial velocity shifts in some of the lines previously used to derive this value. The individual spectra were normalised to a consistent pseudo-continuum by fitting splines through carefully selected (line-free) regions in the vicinity of the spectral features of interest. These regions were originally identified in the mean spectrum of the time-series.

The FEROS data set outlined above is ideal for monitoring the development and evolution of substantial wind activity during each night, and from night-to-night. We are also able to derive information on the longer-term wind behaviour (e.g. year-to-year) from the fact that HD 152408 was included as one of the targets in previous extended monitoring programs carried out by the Landessternwarte Heidelberg. In particular, we have examined nightly spectra taken in 1995, 1996 and 1999 with the Heidelberg Extended Range Optical Spectrograph (HEROS) on the ESO 50-cm telescope at La Silla in Chile. A summary list of these observations is given in Table 2. HEROS provides a resolving power of $\sim 20000$ and a total wavelength range of 3500 to $8600 \AA$, which is covered in two channels (blue: $3540-5600 \AA$ and red: 5800-8600 A). Individual spectrograms have a signal-to-noise ratio $\sim 100$ for 1 hour exposures. The basic strategy of the HEROS campaigns was to secure one spectra per night over several weeks and months, for a broad range of early-type stars. The HEROS spectra of HD 152408 were also reduced semiautomatically in a modified ESO-MIDAS échelle context. 


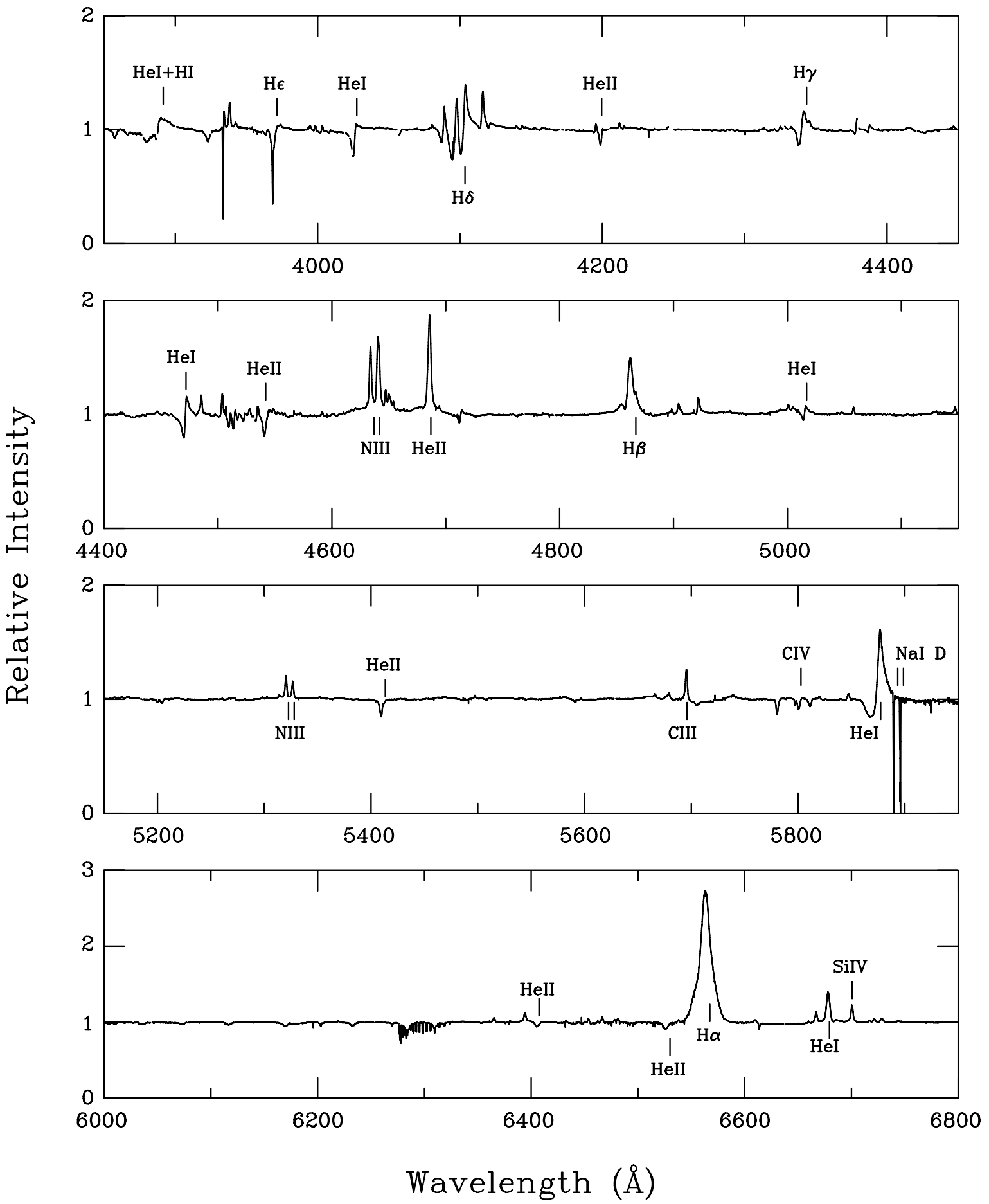

Fig. 1. The mean spectrum of HD 152408 for the FEROS time-series data. Wind activity is analysed in this paper via simultaneous monitoring of absorption and emission fluctuations in several He I and Balmer lines, together with more deep-seated disturbances in the He II absorption and narrow metal emission/absorption lines (e.g. C III, C IV and N III)

\section{Line profile variability}

The time-variable properties of key spectral line profiles in our FEROS data set of HD 152408 from 1999 (Table 2) are discussed here. Prior to that, the mean of the FEROS time-series spectra is shown in Fig. 1. Numerous strong and weak lines are evident, both, in emission and absorption. Our study of stellar wind activity focuses on (i) the
Balmer series of $\mathrm{H} \alpha, \mathrm{H} \beta$, and $\mathrm{H} \gamma$, (ii) the He I lines at $\lambda 3888.65\left(3^{3} \mathrm{~S}-3^{3} \mathrm{P}^{0}\right), \lambda 5875.57\left(2^{3} \mathrm{P}^{0}-3^{3} \mathrm{D}\right)$, and $\lambda 4471.48$ $\left(2^{3} \mathrm{P}^{0}-4^{3} \mathrm{D}\right)$, and (iii) the metal emission and absorption lines due to C III $\lambda 5695.92$, N III $\lambda \lambda 4634-4641$, and C IV $\lambda 5801.31$. The well-developed P Cygni profile of He I $\lambda 5876$ and the substantial $\mathrm{H} \alpha$ emission profile serve as probably the most important lines for a study of the temporal wind behaviour in HD 152408. The blue wings of these lines 

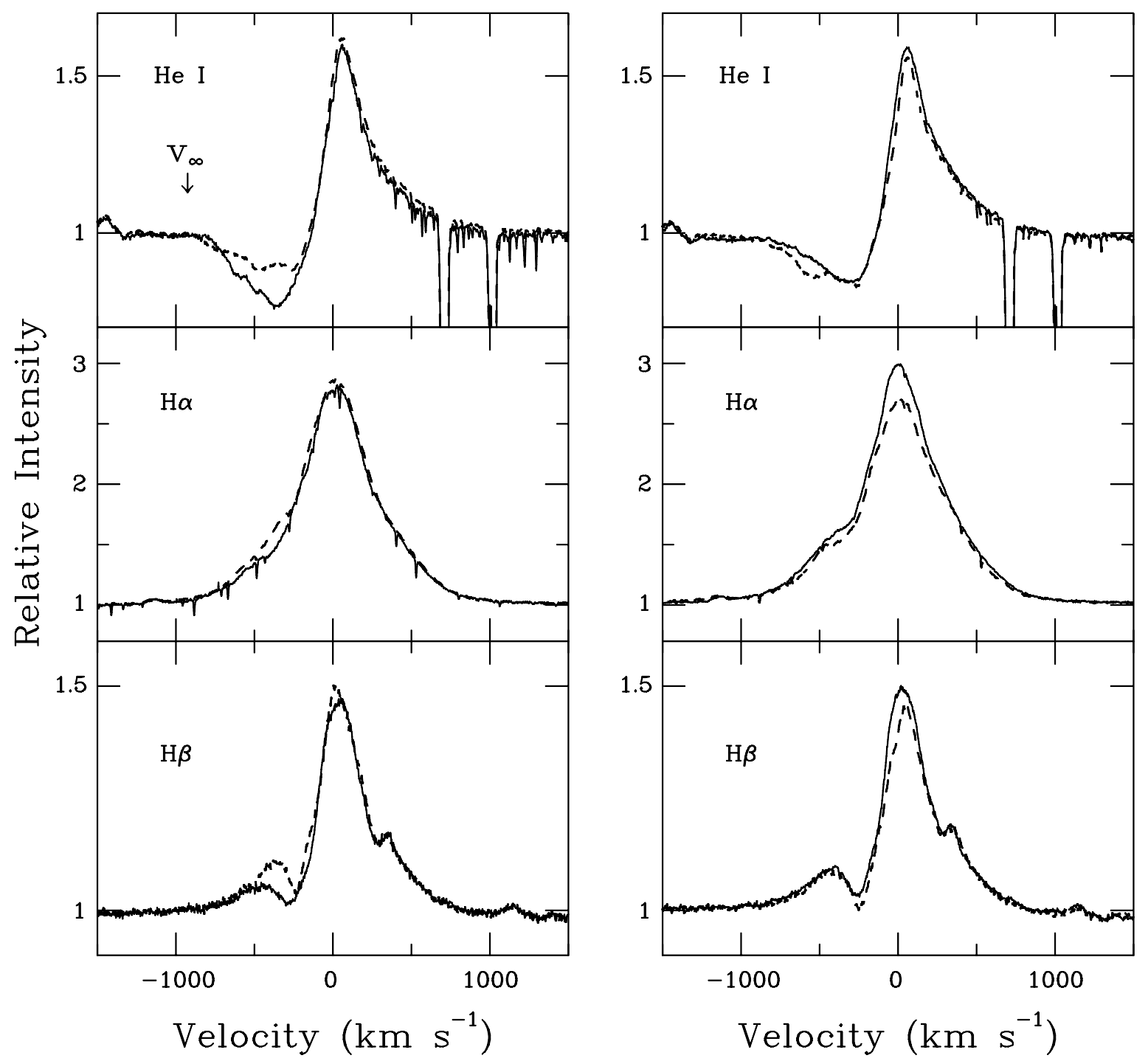

Fig. 2. Variability in the wind-formed lines of HD 152408 takes two principal forms: (i) left-hand panels - localised blue-shifted absorption enhancements are seen in He I $\lambda 5876$, which correspond to reduced flux at the same velocities in the blue wings of $\mathrm{H} \alpha$ and $\mathrm{H} \beta$. (Localised emission features are also present in the red-wing of $\mathrm{H} \alpha$, e.g. Fig. 3.), (ii) right-hand panels - the central emission peak in $\mathrm{H} \alpha, \mathrm{H} \beta$ and He I $\lambda 5876$ can change substantially, mostly within a velocity region of $\sim-200$ to $200 \mathrm{~km} \mathrm{~s}{ }^{-1}$. The terminal velocity, $v_{\infty}$, is marked (upper-left panel). The weak emission feature in the red wing of $\mathrm{H} \beta$ at $\sim 400 \mathrm{~km} \mathrm{~s}$ is due to $\mathrm{N}$ III (and similarly for $\mathrm{H} \gamma$ in Fig. 1)

extend to more than $90 \%$ of the wind terminal velocity derived from saturated UV P Cygni profiles (see Table 1).

Some examples of changes in corresponding He I $\lambda 5876$, $\mathrm{H} \alpha$ and $\mathrm{H} \beta$ line profiles are shown in Fig. 2. These data highlight two fundamental forms of variability:

1. (Fig. 2; left-hand panels) Localised (in velocity space) absorption enhancements, are clearly noted in the blue-ward troughs of the He I profile. Reduced flux is also seen at essentially the same velocities in the blue wings of $\mathrm{H} \alpha$ (and $\mathrm{H} \beta$ ). This absorption effect is examined further below. The absorption depths of the discrete features in He I $\lambda 5876$ may change by up to $\sim 10 \%$ with respect to the mean profile;

2. (Fig. 2; right-hand panels) Substantial changes in the peak and central emission strength are noted in $\mathrm{H} \alpha$, and the total emission equivalent width may vary by up to $\sim 20 \%$. Sympathetic fluctuations are also evident in $\mathrm{H} \beta$ and $\mathrm{He}$ I $\lambda 5876$. The $\mathrm{H} \alpha$ variations are most prominent in the central regions of the line profiles.

In order to examine systematic and progressive changes in the optical line profiles, the FEROS time-series data are shown as grey-scale representations in Fig. 3 (a; Balmer lines and b; He I lines). To enhance the contrast in these images, and following convention, the respective mean line profile is subtracted from each spectrum in the time-series. (The mean profiles used are shown in the bottom panels for each case in Fig. 3.) Regions of excess absorption with respect to the mean therefore appear darker, while lighter shades correspond to intervals when the local flux was greater than the mean value. Additionally, in order to 

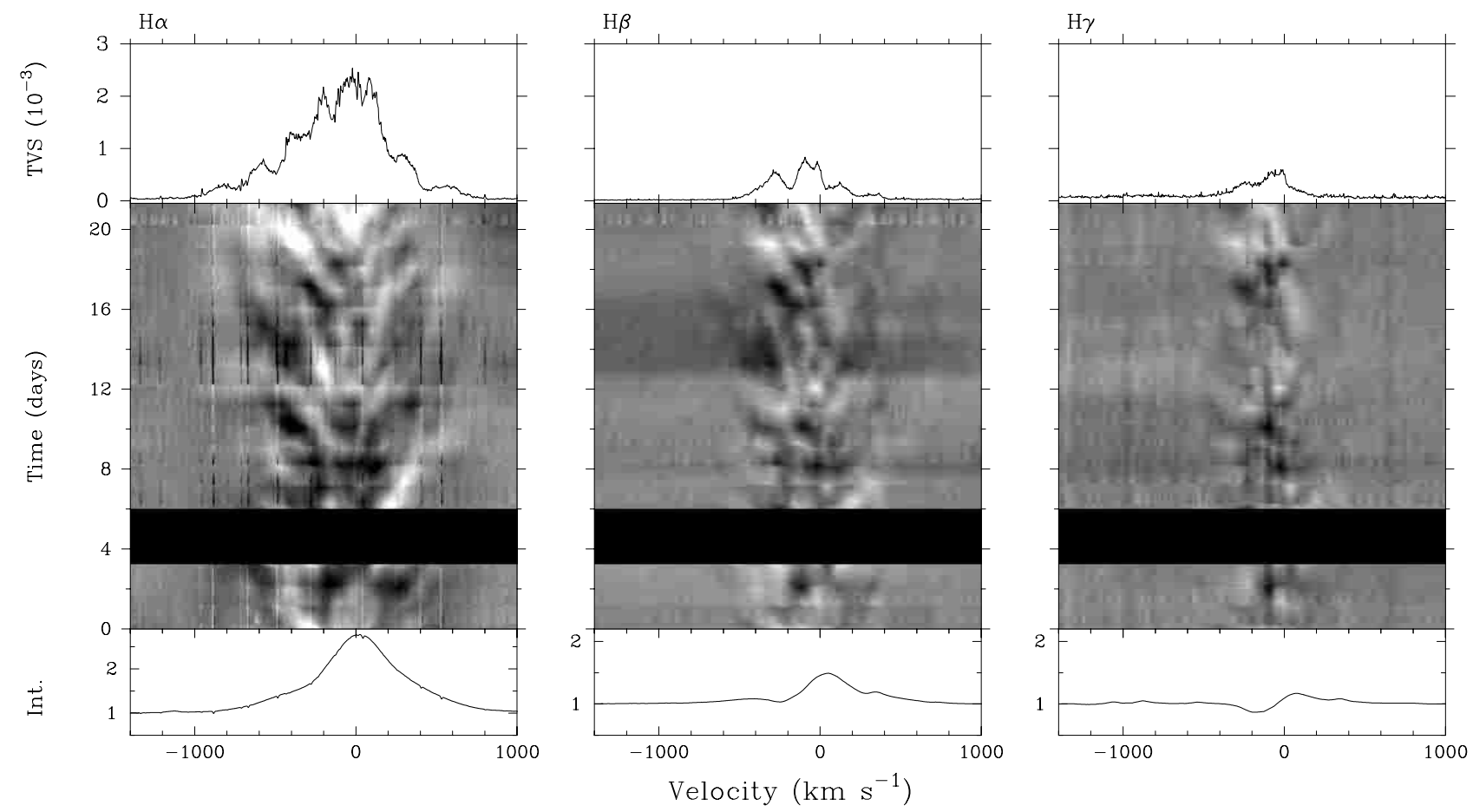

Fig. 3. a) Grey-scale representations of variability in the FEROS Balmer lines of HD 152408. Darker shades indicate regions of enhanced absorption (reduced flux) with respect to the mean profile (which is shown below each grey-scale image). The scales for the images correspond to differences from the mean of $+5 \%$ (white) and $-5 \%$ (black). The temporal variance spectrum (TVS, Sect. 3) is shown above each dynamic image. The level of significance in the TVS for a probability of $95 \%$ is $\sim 310^{-5}$

quantify the extent of variability as a function of velocity, we computed the temporal variance spectrum (TVS) for each line profile in Fig. 3 (see e.g. Fullerton et al. 1996):

$$
(T V S)_{i}=\sigma_{0}^{2} \frac{1}{N-1} \sum_{j=1}^{N}\left(\frac{S_{i j}-\bar{S}_{i}}{\sigma_{j c} \sqrt{S_{i j}}}\right)^{2}
$$

where $S_{i j}$ is the normalized intensity of the $i$ th pixel in the $j$ th spectrum, $\bar{S}_{i}$ is the weighted mean of the normalized intensity, $\sigma_{j c}$ is the inverse of $S / N$ of spectrum $j$ measured in a continuum band, and $\sigma_{0}^{2}=\left[\frac{1}{N} \sum_{j=1}^{N} \sigma_{c j}^{-2}\right]^{-1}$. The TVS results are also shown in Fig. 3, above each greyscale image.

The illustrations in Fig. 3 demonstrate that the stellar wind of HD 152408 is continuously variable over the $\sim 21$ days of FEROS observations. The line profile behaviour revealed is clearly not erratic, but is instead organised into sequential localised episodes of enhanced and/or reduced flux, which migrate in velocity as a function of time. The discrete features develop remarkably slowly, and typically evolve between $\sim 3-8$ days (see further discussion below). These time-scales are substantially longer than those reported, for example, by Eversberg et al. (1998) for the case of small-scale structure in the O4 supergiant $\zeta$ Pup. The systematic variations in the $\mathrm{H} \alpha$ profiles (Fig. 3) are noted at blue-ward and red-ward velocities, and the former are mimicked in $\mathrm{H} \beta$ and $\mathrm{H} \gamma$. A substantial enhancement is noted in the central $\mathrm{H} \alpha$ emission strength towards the end of the time series (see also Fig. 2). It is fairly clear from the grey scale images that the discrete absorption features seen in the short-ward extended troughs in He I $\lambda 5876$ correspond very closely to episodes of reduced flux in the blue wings of the Balmer lines. We tested this relation (and those between other velocity regimes and line profile components) by computing co-variance matrices from individual time-series spectra. The procedure allows the variable flux at a given velocity bin to be directly correlated against every other velocity bin across a line profile. The results from the co-variance analysis are shown in Fig. 4 as two-dimensional grey-scale representations, where stronger positive correlations are flagged by whiter shades. A very tight correlation is present between changes in the He I $\lambda 5876$ blue absorption trough and the $\mathrm{H} \alpha$ blue wing. This result confirms the notion that substantial wind structure is seen in absorption in $\mathrm{H} \alpha$. Despite initial appearance in Fig. 3a, there is no localised correspondence between changes in the blue and red wings in $\mathrm{H} \alpha$ (or blue-ward and red-ward velocities in He I $\lambda 5876$ ); the brighter regions in the upper-left panel in Fig. 4 relate to the increase in peak emission strength seen towards the end of the FEROS data set (see also Fig. 3a). In this case the increase in $\mathrm{H} \alpha$ central emission extends simultaneously to the blue and red wings, but not to such high velocities as the discrete migrating features. Finally, we note in Fig. 4 (bottom-right panel) that there is some correlation between fluctuations in the $\mathrm{H} \alpha$ red emission and the (much weaker) He I $\lambda 5876$ emission component.

We do not find any unique relation in terms of a time-lag between the occurrence of the episodic blue-ward 

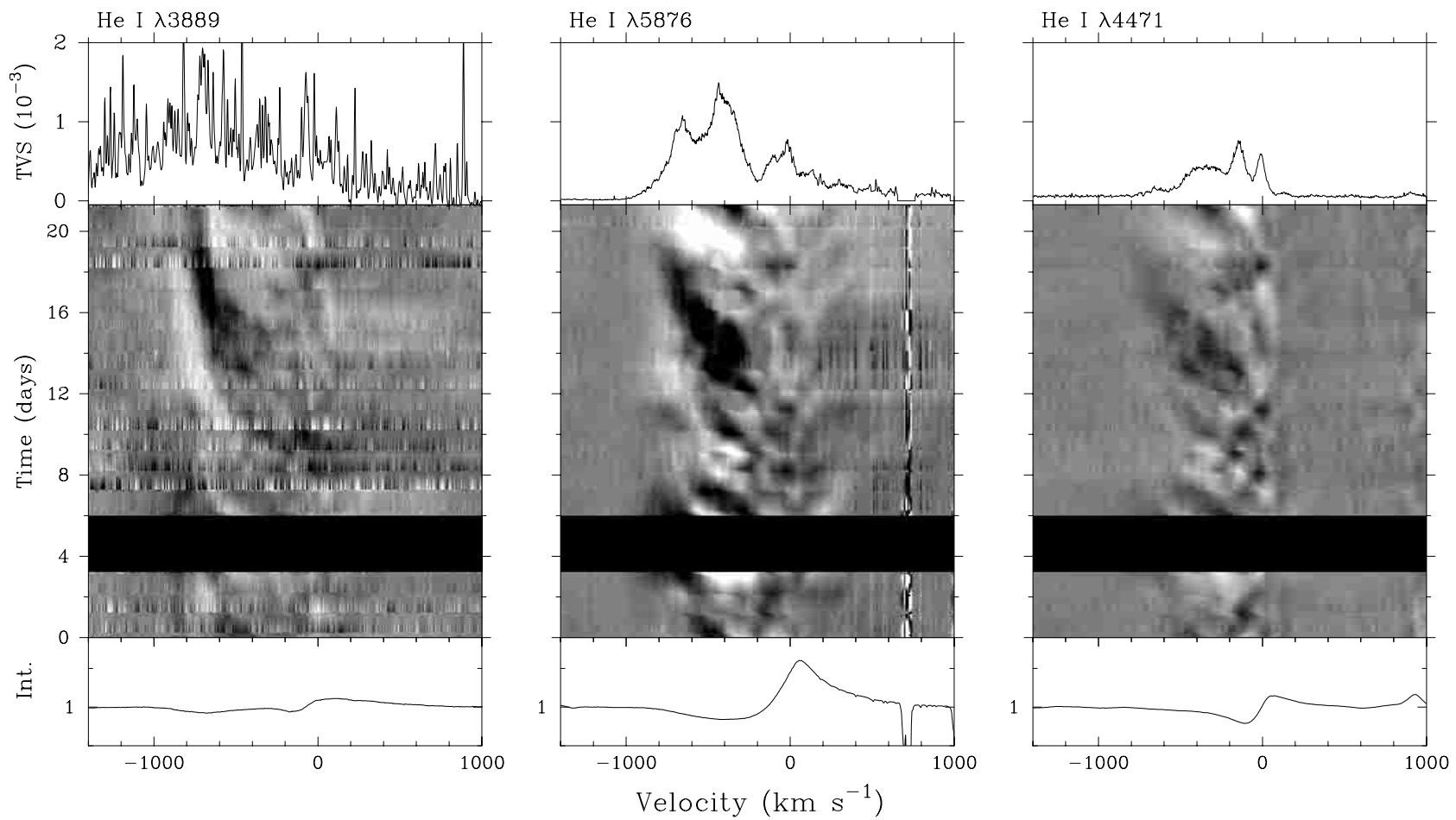

Fig. 3. b) Same as Fig. 3a except for the He I lines at $\lambda 3889, \lambda 5876$ and $\lambda 4471$

migrating features and the red-ward features. A lag may be expected, for example, as a given structure that is rooted to stellar surface is carried through the line-of-sight at the rotation rate. A unique lag of a reasonable fraction of the rotation rate (e.g. $\sim 5$ days or more) cannot be identified in our data. The analysis is complicated by the fact that multiple features may be present at a given time, which prevents an unambiguous connection between a given blue-ward and red-ward feature. (Furthermore, we note below that the accelerations of the blue and red features are in fact different in any case.)

The TVS of $\mathrm{H} \alpha$ in Fig. 3a is clearly asymmetric such that the variability in the blue wing is more extended in velocity (by more than $300 \mathrm{~km} \mathrm{~s}^{-1}$ ) than in the red wing. (The mean $\mathrm{H} \alpha$ profile is by contrast highly symmetric despite some blending due to He II $\lambda 6560.2$.) This asymmetry in the variance characteristics is also apparent to a similar degree in $\mathrm{H} \beta$ (Fig. 3a). These results are consistent with an empirical picture where the changes in the blue $\mathrm{H} \alpha$ wing are dominated by the effects of episodic absorption features, which arise from wind structure localised along the line-of-sight to the stellar disc. In contrast, the red-ward $\mathrm{H} \alpha$ wing is distorted by emission humps due to large-scale structure moving away from our line-of-sight. A blue-to-red asymmetry in the $\mathrm{H} \alpha$ TVS is expected in this scenario since the overall emission profile is produced by gas occupying a considerably greater volume than the gas responsible for absorption features.

The He I TVS results point to blue-ward variability extending down to very low velocities (less that $-50 \mathrm{~km} \mathrm{~s}^{-1}$; e.g. He I $\lambda 4471$ in Fig. 3b). It is perhaps unusual to note the degree of structure in the HeI TVS, which may in part be due to the relatively strong absorption enhancement noted between $\sim 11$ to 20 days into the time-series.

There is a surprising but clear discrepancy between the observed time-variable velocity behaviour of the blueward migrating features compared to the red-ward ones. The velocities of 10 blue-ward features in He I $\lambda 5876$ and 6 red-ward features in $\mathrm{H} \alpha$ were measured as a function of time, directly from the grey-scale images using a simple cursor method (e.g. Fig. 3). The results are plotted in Fig. 5. All the blue-ward features have broadly the same acceleration rates and the same is true for the red-ward propagating features; however, the blue-ward features accelerate faster than the red-ward ones. The mean accelerations in Fig. 5 are $\sim 185 \mathrm{~km} \mathrm{~s}^{-1}$ day $^{-1}$ and $85 \mathrm{~km} \mathrm{~s}^{-1}$ day $^{-1}$ for blue and red cases, respectively. Over the same time interval the maximum velocity reached by the red features is only about $50 \%$ of that reached by the blue-ward features. This result is of course consistent with the TVS discussed earlier.

It seems unlikely therefore that the discrete features in the line profiles are due to the motion of mass-conserving blobs travelling in the wind, since they would be expected to follow trajectories of varied inclinations to the lineof-sight to the observer, and hence provide a range of projected velocities and accelerations. Furthermore, the accelerations of these features are typically only $\sim 15$ to $20 \%$ of the values expected for " $\beta=1$ "-type velocity laws in steady-state wind models. Sampled at $\sim 0.5 v_{\infty}$ 


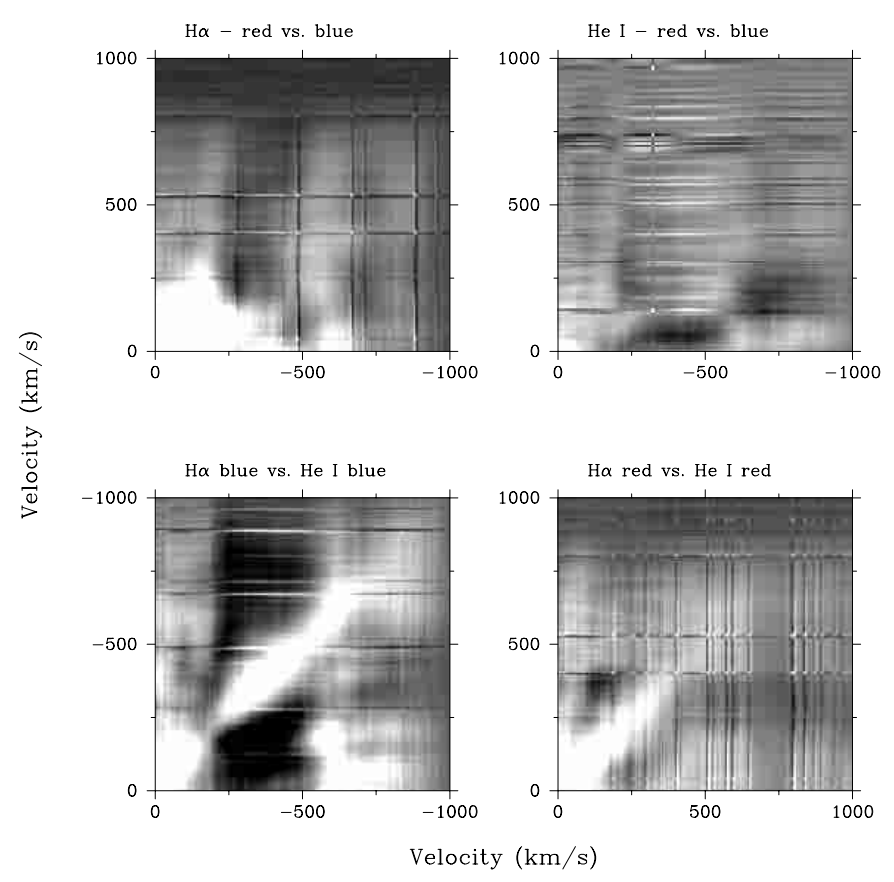

Fig. 4. Grey-scale representations of the co-variance in the FEROS data between changes in (i) the red and blue wings of $\mathrm{H} \alpha$ (top-left), (ii) the $\mathrm{H} \alpha$ blue wing and $\mathrm{He}$ I $\lambda 5876$ absorption trough (bottom-left), (iii) the red emission and blue absorption components of He I $\lambda 5876$ (top-right), and (iv) the $\mathrm{H} \alpha$ red wing and He I $\lambda 5876$ red emission component (bottom-right). A positive linear correlation is flagged as white shades in these diagrams

the accelerations of the absorption features in the He I lines are also $\sim 30$ to $40 \%$ less than recorded for "classical" DACs in the UV resonance lines (see e.g. references in Sect. 1). The inference is that localised variability in the $\mathrm{He}$ I absorption troughs and $\mathrm{H} \alpha$ wings betrays the effects of very slowly evolving perturbations ( over several days), through which wind material flows. We postpone further discussion of the asymmetry recorded in Fig. 5 until Sect. 8.

\subsection{The Hel discrete absorption episodes}

The three He I lines shown in Fig. 3b provide a fine opportunity to trace and compare wind material from very low velocities and radial depths in the wind (i.e. He I $\lambda 4471$ ) to progressively greater distances from the stellar surface (i.e. He I $\lambda 5876$ and He I $\lambda 3889$ ). Though several different episodes are evident between these lines, the strongest and best resolved cases are seen after $T \sim 11$ days in Fig. $3 b$. To derive simple measures of the relative properties of structure in these lines, we analysed the mean-subtracted spectra in Fig. 3b by (least squares) fitting them with a Gaussian absorption profile to determine the central velocities and relative overall strengths. In most cases (despite some strong blending effects) the model Gaussian profiles provide good matches to the difference spectra.

Results from the profile fits are shown in Fig. 6 for the episodic features recorded between $T \sim 11$ to 21 days in

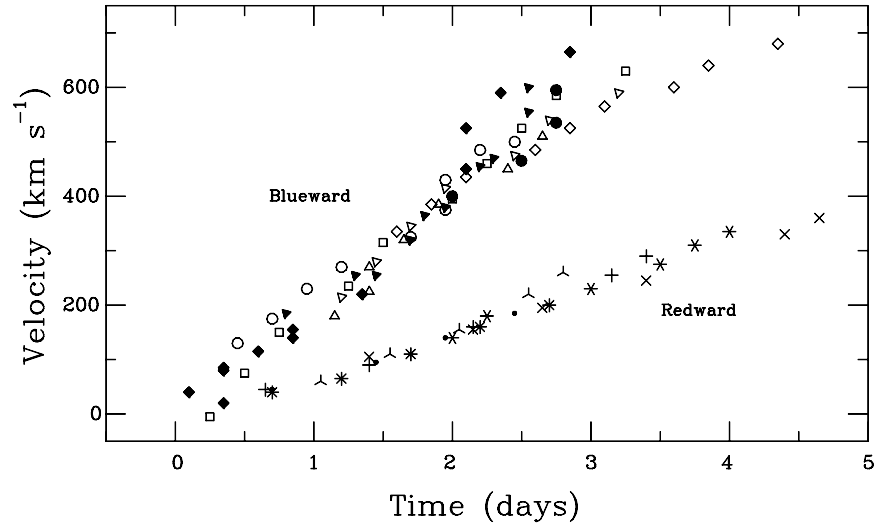

Fig. 5. The accelerations of 10 separate discrete features evident at blue-ward velocities in the absorption trough of He I $\lambda 5876$ (closed symbols) are compared to those of 6 red-ward migrating features in $\mathrm{H} \alpha$ (skeletal symbols and dots)

He I $\lambda 3889$, He I $\lambda 5876$ and He I $\lambda 4471$. The figure describes the properties of 3 separate features (labelled "a", "b" and "c" in the caption). The linear accelerations of features "a" and "b" is fairly consistent between the different He I lines, but feature "c" appears to evolve slower in He I $\lambda 3889$ than in He I $\lambda 5876$ (see also Fig. 3b).

It is also interesting to compare the relative strength of the discrete features in Fig. 5 as a function of central velocity. The episodes seen in He I $\lambda 4471$ have a peak strength at lower velocity than in He I $\lambda 5876$; in the latter case the absorption strength rises until a velocity of $\sim-420 \mathrm{~km} \mathrm{~s}^{-1}$, and the features subsequently fade away. In greater contrast, the same features continue to strengthen in He I $\lambda 3889$ up to $\sim-600 \mathrm{~km} \mathrm{~s}^{-1}$. These simple measurements sample regions well blue-ward of the moderate-to-weak emission components of the He I lines, and are unlikely to be grossly affected by any underlying emission changes.

\subsection{Current overview of time-dependent wind properties}

Before proceeding further, the principal characteristics of the time-variable behaviour of optical lines in HD 152408 derived in this section are briefly summarised here. The stellar wind of this massive luminous star, which has an average projected rotation velocity, is strongly affected by organised inhomogeneities. In contrast to small-scale features seen in the optical lines of WR and some $\mathrm{O}$ stars, these structures evolve very slowly over several days and represent large-scale perturbations. They are revealed by localised absorption enhancements in the He I lines, and with close correspondence - by discrete regions of reduced flux in the blue wings of $\mathrm{H} \alpha$. The structures are also diagnosed by emission humps in the red wing of $\mathrm{H} \alpha$. The discrete features in the line profiles generally accelerate more slowly than "classical" DACs seen in the ultraviolet resonance lines of $\mathrm{OB}$ stars. An important constraint 

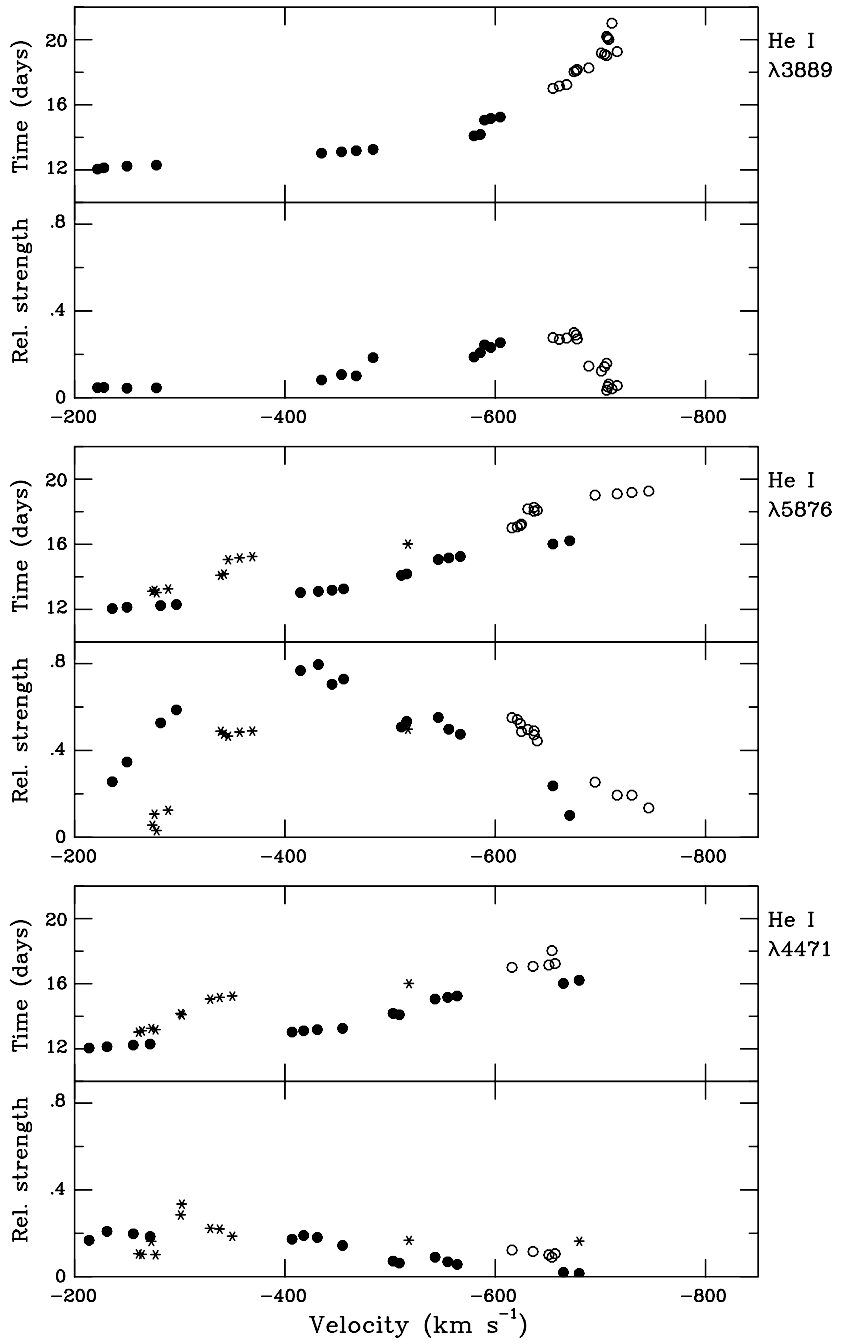

Fig. 6. The central velocities and relative strengths (in arbitrary units) of 3 strong discrete absorption features in the (FEROS) HeI $\lambda 3889, \lambda 5876$ and $\lambda 4471$ line profiles are compared. The results are based on Gaussian profile fitting (Sect. 3.1). The closed circles, asterisks, and open circles in this figure correspond to features labelled a), b) and c), respectively, in Sect. 3.1

is that red-ward migrating features typically accelerate $\sim 50 \%$ slower than the blue-ward features.

Extended FEROS spectroscopy, with simultaneous time-series coverage of He I and Balmer lines, has permitted us to demonstrate absorption effects at blue-ward velocities in the Balmer lines. This is perhaps an unexpected result for an Of or WR star since emission processes normally dominate in these lines as a result of recombinations from a higher ionization state, which are followed by radiative de-excitations. If the wind is extremely opaque in hydrogen, however, there would be detailed balancing between photoionization out of, and recombination back into the $\mathrm{H}^{0}$ ground state. In the dense wind case of HD 152408 the line formation mechanism in the wind is therefore such that there is a substantial population in the lower (second) level of hydrogen, which then effectively becomes the ground state and the line behaves as a scattering line. This is in fact the transition that yields the He I $\lambda 5876 \mathrm{P}$ Cygni profile for this star. In this respect, some parallels may be drawn, for example, between the very dense and relatively slow wind of HD 152408 and that of the luminous hypergiant P Cygni (see e.g. the wind model of Drew 1985).

\section{Search for modulated wind activity}

We discuss next whether the stellar wind variations described in Sect. 3 are repetitive or quasi-cyclic. A rigorous time-series analysis of the line profile fluctuations in the FEROS data set was carried out using the discrete Fourier transform methods described, for example, by Gies \& Kullavanijaya (1988) and Baade (1988). We used the iterative CLEAN algorithm (Roberts et al. 1987) to deconvolve the features of the window function from the discrete Fourier transform. The results discussed here have been CLEANed for 200 iterations and a gain of 0.2 .

The CLEANed Fourier transforms for He I $\lambda 5876, \mathrm{H} \alpha$ and $\mathrm{H} \beta$ are illustrated in two-dimensional image representations in Fig. 7. The dark horizontal bands in these images represent peak power signals of the same frequency over an extended velocity range in the line profile. For each spectral line in Fig. 7, the right-hand-panel shows power as a function of frequency, summed over the entire line profile (i.e. a mean periodogram). The mean spectrum of the time-series is plotted in the lower panel. The images in Fig. 7 provide some evidence for modulated activity at two frequencies, with a reasonable degree of consistency between the 3 spectral lines. In decreasing order the dominant frequencies are: $0.131 \pm 0.030 \mathrm{~d}^{-1}$ and $0.263 \pm 0.032 \mathrm{~d}^{-1}$ in He I $\lambda 5876 ; 0.139 \pm 0.027 \mathrm{~d}^{-1}$ and $0.256 \pm 0.033 \mathrm{~d}^{-1}$ in $\mathrm{H} \alpha ; 0.122 \pm 0.036 \mathrm{~d}^{-1}$ and $0.258 \pm$ $0.019 \mathrm{~d}^{-1}$ in $\mathrm{H} \beta$. (The errors represent the half-width at half-maximum of the periodogram peaks.) The mean of these frequencies correspond to periods of $P_{1}=7.67$ and $P_{2}=3.86$ days (with an uncertainty of $\sim 20 \%$ ). The dominant signal, $P_{1}$, is almost exactly $2 \times P_{2}$ and the power in $P_{1}$ extends over a larger velocity range in all lines, including slightly red-wards in $\mathrm{H} \alpha$.

A quasi-cyclic modulation in the absorption enhancements is more striking in He I $\lambda 5876$, and perhaps better defined for a period of $\sim 7.7$ days than 3.9 days. These two (dominant and harmonic) periods are approximately 2.5 and 5.0 times the estimated maximum rotation period of HD 152408 (Table 1). The projected rotation rate is very average for its spectral type, and thus the inclination of the rotation axis to the line-of-sight is unconstrained. We cannot therefore uniquely establish (or rule out) strict rotational modulation of the wind in HD 152408. The timescales of the repetitive behaviour documented in Fig. 7 are rather long and of course correspond to the slow velocity evolution of individual episodic features in the line profiles. In contrast, the characteristic radial wind flow time of HD 152408 (i.e. $\sim R_{\star} / v_{\infty}$ ) is $\sim 6.5$ hours, which is $\sim 28$ times the dominant modulation period $P_{1}$. Since the modulation time-scales associated with the large-scale wind structures are significantly greater than the wind 
He I $\lambda 5876$

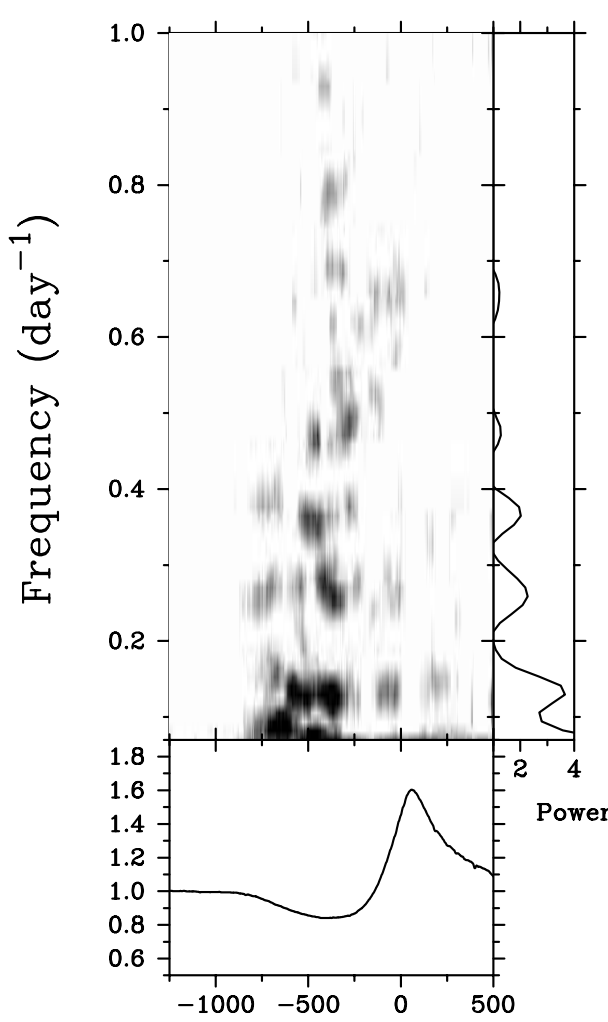

$\mathrm{H} \alpha$

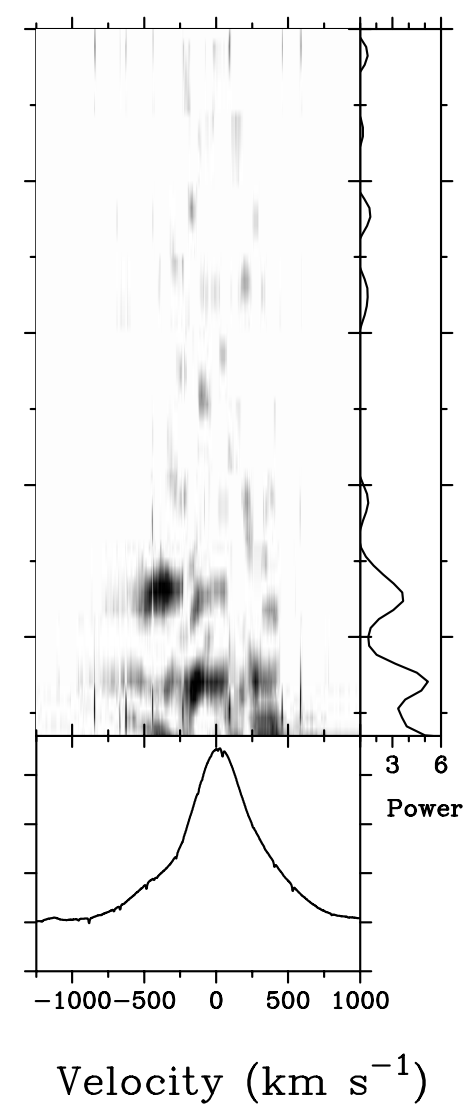

$\mathrm{H} \beta$

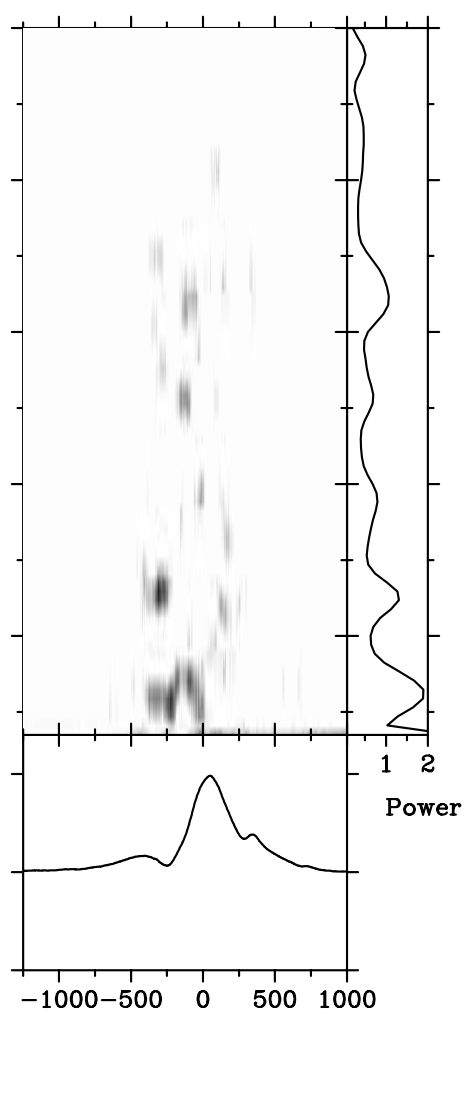

Fig. 7. Results from the discrete Fourier transform analysis of FEROS data for He I $\lambda 5876, \mathrm{H} \alpha$, and $\mathrm{H} \beta$. The grey-scale images show power as a function of position in the line profile, and the frequency distribution of power summed over each line profile is shown in the right-hand panels. The mean line profiles are shown in the bottom-panels. We identify the dominant frequency at $\sim 0.13 \mathrm{~d}^{-1}$ and its harmonic at $\sim 0.26 \mathrm{~d}^{-1}$

flushing time over the optical line formation region, it is very unlikely that the wind structures diagnosed in our optical data are due to processes entirely intrinsic to the wind. The perturbations likely arise as a result of inhomogeneities rooted at the stellar surface and are controlled to some degree by the rotation rate of the star. This is the simplest phenomenological picture for generating time-scales of $\sim$ several days in the winds of $\mathrm{O}$ and early-B supergiants. The substantial "underlying" wind of HD 152408 is also expected to be riddled by small-scale structure that arises from the action of the intrinsic linestability mechanism on very short time-scales and lengths. Though we have not directly detected small-scale structure in our spectroscopy, it would act to disrupt the development of large-scale features (e.g. Owocki 1999). At minimum, this interaction would lead to decreased coherence in the observed wind modulations and generally more ambiguous evidence for repeatability (e.g. in Fig. 3). Indeed, it is surprising to witness systematic patterns in the wind-formed line profiles of HD 152408 that persist for $\sim 20$ days and possibly much longer (e.g. Sect. 6), given that the "underlying" wind is expected to be so dynamically unstable.

To investigate further the quasi-periodic behaviour identified in Fig. 7 we extended the Fourier analysis to determine for each position in the spectral line, the semiamplitude and phases for the best-fit sinusoids for the two periods, $P_{1}$ and $P_{2}$. The results are illustrated in Fig. 8 for He I $\lambda 5876$, He I $\lambda 4471$ and $\mathrm{H} \alpha$. The semi-amplitude is expressed as a percentage of the continuum, and is approximately twice the amplitude of the power spectrum at the frequency of a detected period. The phase is determined from the first spectrum of the FEROS time-series and the distribution of phase constant runs from $-\pi$ to $\pi$ (Fig. 8). The phase change as a function of velocity shows how the sinusoid of a detected period moves across the line profile. For each spectral line the mean line profile is plotted in the bottom panel for reference. The point-to-point scatter in the phase constant across the line is perhaps slightly lower for $P_{1}$ than $P_{2}$, since the former is more convincing in our data.

Despite initial appearance, it is important to note that the phase as a function of velocity for $P_{1}$ in Fig. 8 is not genuinely "bowed". For the case of $P_{1}$ the phase is most advanced in the short-ward absorption trough at $\sim-315 \mathrm{kms}^{-1}$, in both He I lines. Blue-ward of this position the phase increases approximately linearly toward the wings of the lines, in the direction of the propagating absorption features (Fig. 3). The apparent increase in phase red-ward of $\sim-315 \mathrm{~km} \mathrm{~s}^{-1}$ is artificial, 

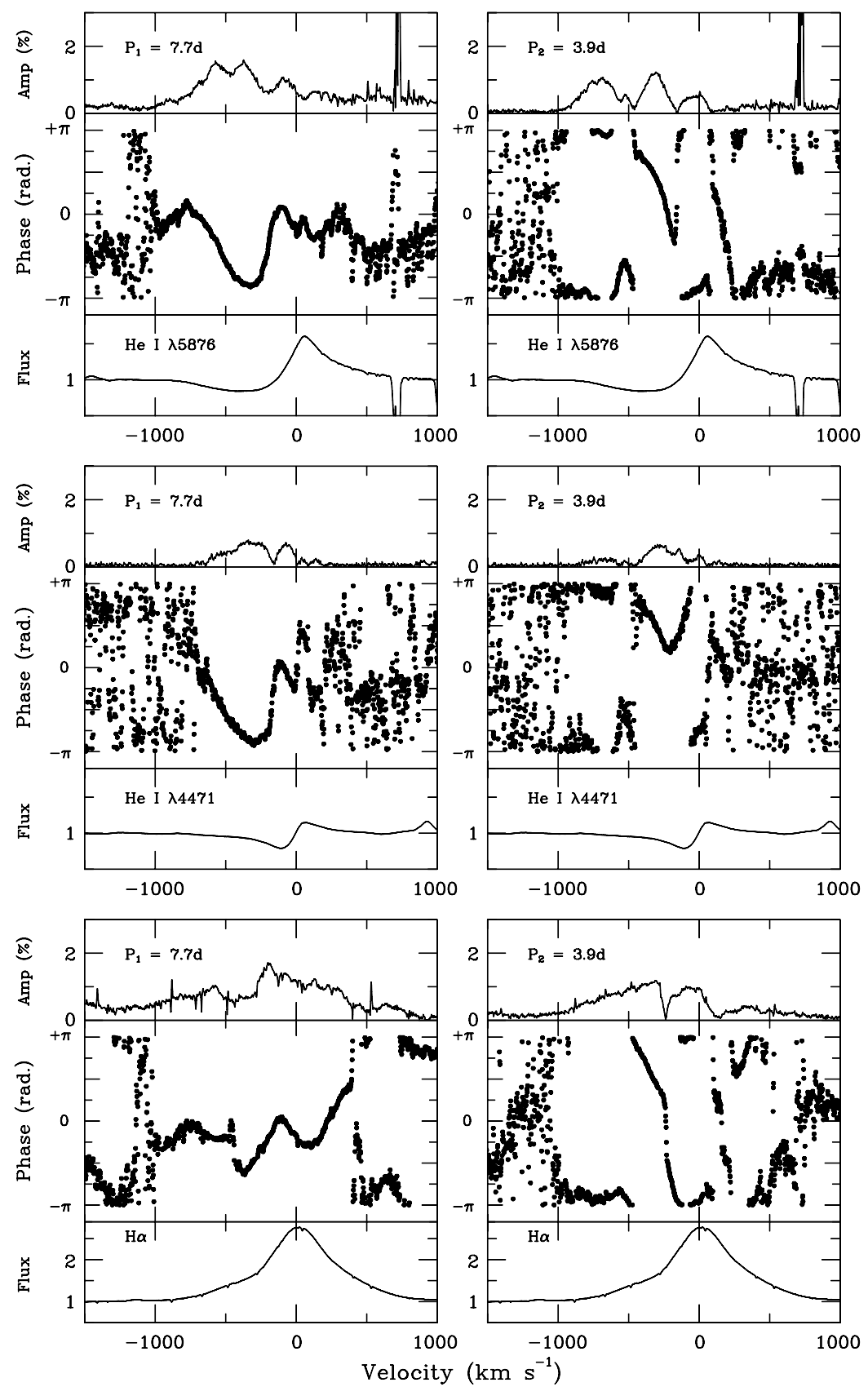

Fig. 8. The complex phase and sinusoidal (\%) half-amplitude is shown as a function of velocity for He I $\lambda 5876$, He I $\lambda 4471$ and $\mathrm{H} \alpha$ for both the periods identified in HD 152408 ( $P_{1}$ on the left-hand side, and $P_{2}$ on the right-hand side). The corresponding mean FEROS line profiles are also shown below each phase diagram. Note, that despite its appearance here, the distribution of phase constant with velocity is not genuinely "bowed" (see Sect. 4)

however, and not due to the propagating localised features. The much steeper rise in phase between $\sim-315$ to $\sim-100 \mathrm{~km} \mathrm{~s}^{-1}$ is instead due to a "connection" made in the phasing algorithm between the dominant low velocity region of each episode (at $\sim-300 \mathrm{~km} \mathrm{~s}^{-1}$ ) and the initial appearance (between rest velocity and $-100 \mathrm{~km} \mathrm{~s}^{-1}$ ) of the subsequent feature. The contrived link between the low velocity regions of 2 separate epsiodic features results in the impression of increased redward phase in the diagram in Fig. 8. To a lesser extent this phase behaviour is also noted at blue-ward velocities in $\mathrm{H} \alpha$. Furthermore, inspection of the grey-scales in Fig. 3 and the velocity versus time behaviour in Fig. 5, provides no convincing evidence for "bowed" structures of the kind noted in HD 64760 (i.e., features extending simultaneously to lower and higher blue-shifted velocities; Fullerton et al. 1997). It is indeed likely that any azimuthal extent to the wind structure in a moderate rotator such as HD 152408 will not be so pronounced, particularly in the inner-wind region sampled in our optical data (see Sect. 8).

\section{Deep-seated disturbances}

We have demonstrated in the previous section that the variability seen in the Balmer and He I wind lines spans a wide velocity range, from almost the rest velocity to the terminal velocity. If the structures responsible for the wind activity are indeed anchored at or close to the stellar surface then a natural next step would be to examine time-dependent behaviour in weak photospheric lines. 

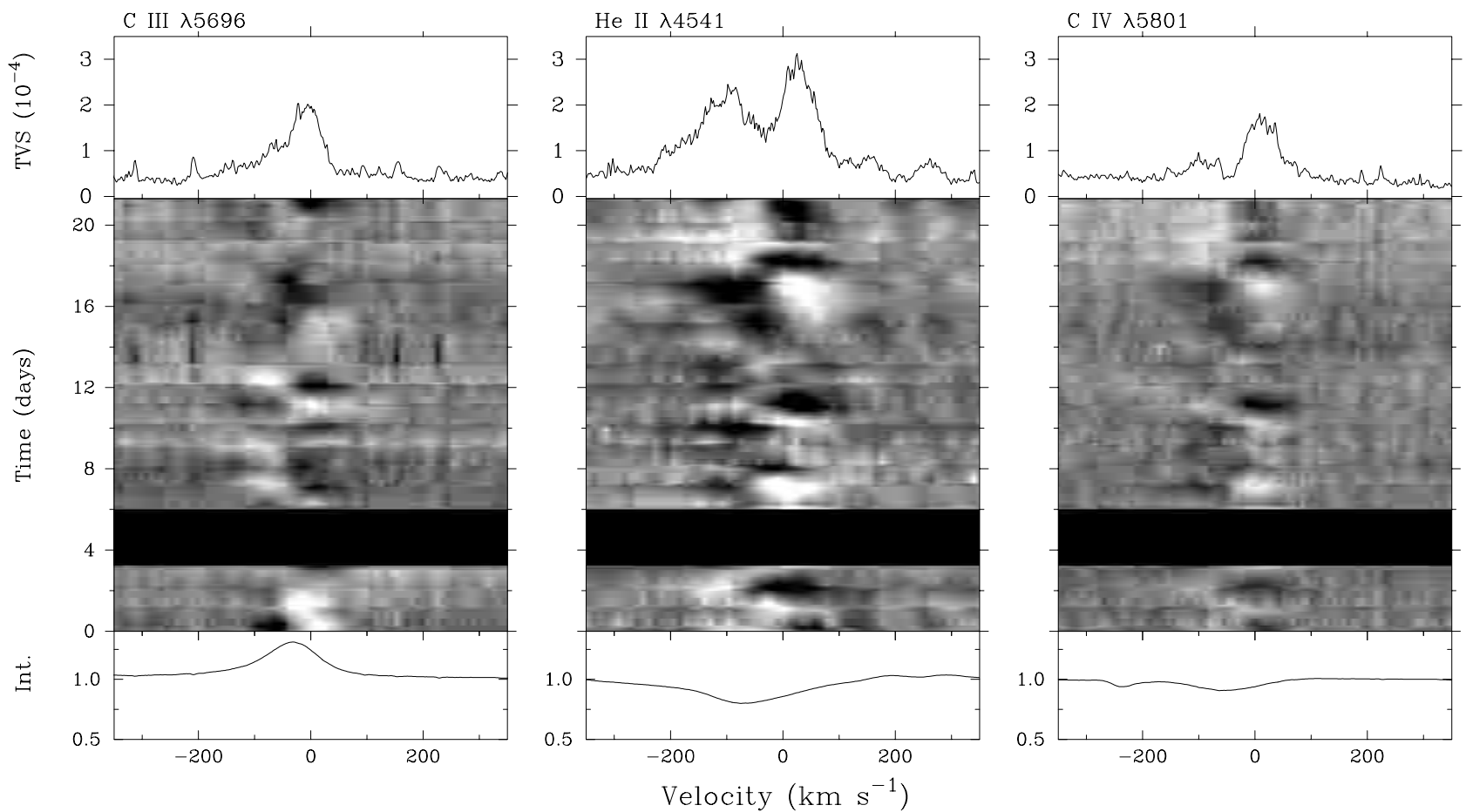

Fig. 9. Grey-scale representations of variability in the FEROS data of the weak metallic line due to C III $\lambda 5696$, and the weak absorption troughs of He II $\lambda 4541$ and C IV $\lambda 5801$. The temporal variance spectrum (TVS; Sect. 3 ) is shown above each image. The dynamic images show differences from the mean profiles (shown in the bottom panels) of $+3 \%$ (white) to $-3 \%$ (black)

Unfortunately, in the dense-wind case of HD 152408 there are essentially no "pure" photospheric features in our optical spectrograms, i.e. measurable lines that are totally unaffected by stellar wind contamination. We can, however, consider slightly asymmetric (weak) C IV and He II absorption lines and weak metallic emission features to diagnose any evidence for deep-seated activity very close to the photosphere of HD 152408.

A grey-scale representation of changes in our FEROS time-series data of C III $\lambda 5696$ (emission), He II $\lambda 4541$ (absorption) and C IV $\lambda 5801$ (absorption) is shown in Fig. 9. There is a good general agreement between the variations evident as a function of time in the shallow absorption troughs in Fig. 9, and also with some of the principal stellar wind events seen in $\mathrm{He}$ I and $\mathrm{H} \alpha$ (cf. Fig. 3). In particular the strong absorption enhancement seen in He I between $T \sim 13$ to 18 days is also partially noted at low velocities (less than $-200 \mathrm{~km} \mathrm{~s}^{-1}$ ) in absorption in He II $\lambda 4541$ and C IV $\lambda 5801$. There are also line profile disturbances centred around zero velocity in these two lines seen near the start of the time-series $(T<3$ days $)$ and towards the end of the observing run $(T>18$ days), which coincide with propagating wind episodes in He I (Fig. 3b).

Despite the wind effects noted above, the absorption line profiles of C IV $\lambda \lambda 5801,5812$ are generally fairly symmetrical and provide the best opportunity to examine activity in layers closest to the photosphere. The grey-scale image in Fig. 9 suggests some evidence for continuous changes in C IV during each night. (This is also true for He II $\lambda 4541$ absorption.) The variability princially takes the form of radial velocity shifts (see e.g. Fig. 10), which were quantified here using least squares Gaussian-profile fitting. A Fourier time-series analysis of the CIV central absorption velocity versus time results indicates peaks in the power signals at a period $\sim 1.45$ days, and less significantly at $\sim 2.30$ days. (Since the beat period between these periods is $\sim 0.9$ days, the $\sim 2.3$ day period might be an alias.) A grey-scale representation of the individual quotient (with respect to the mean) CIV $\lambda 5801$ profiles is shown in Fig. 10, where the data are phased on the $\sim 1.45$ day period (with phase zero arbitrarily set to the first spectrogram in the time-series). The measured central radial velocities are also shown in Fig. 10 phased on this time-scale. The maximum amplitude of the velocity changes is $\sim 25 \mathrm{~km} \mathrm{~s}^{-1}$ and the mean full-width at halfmaximum of the profiles is $\sim 130 \mathrm{~km} \mathrm{~s}^{-1}$. We cannot detect the motion of any sub-features in the weak troughs. The radial velocity shifts reported here may indicate that HD 152408 is pulsating, possibly in the radial fundamental mode. For an estimated mass of $30 M_{\odot}$ (e.g. models in Sect. 7), the pulsational $Q$-value $=P\left(M / M_{\odot} /\left(R / R_{\odot}\right)^{3}\right)^{\frac{1}{2}}$ is $\sim 0.044$. This value and the observed period, $\mathrm{P}$, are fairly consistent with the radial pulsation parameters predicted for evolved supergiants in the $60 M_{\odot}$ initial mass models of Lovy et al. (1984). Alternatively, however, the C IV and He II absorption variability may be due to radial perturbations as a result of "strange-mode instabilities", which are predicted for massive stars located in the upper-left region of the H-R diagram (e.g. Kiriakidis et al. 1993). The ratio of $\left(L_{\star} / L_{\odot}\right) /\left(M_{\star} / M_{\odot}\right)$ for HD 152408 is $\sim 210^{4}$ and 


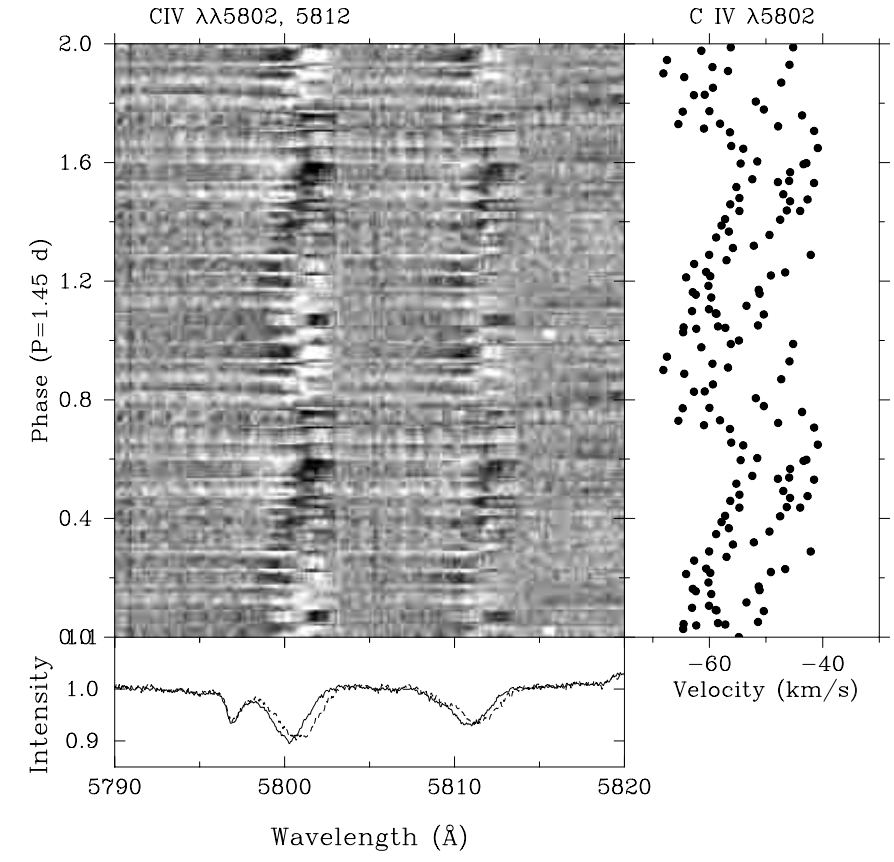

Fig. 10. The grey-scale image shows individual C IV $\lambda \lambda 5801$, 5812 absorption profiles (quotients with respect to the mean profile) phased on the 1.45-day period, and plotted over two cycles (Sect. 5). The corresponding heliocentric radial velocities for the $\lambda 5801$ component are shown in the right-hand panel. A pair of line profiles is shown in the panel below the grey-scale image. The stable feature at $\lambda 5797$ is a diffuse interstellar band (DIB)

is thus in excess of the $\sim 10^{4}$ lower limit quoted by Glatzel (1999), above which strange modes are predicted. A more intensive, higher-resolution, multi-site data set covering about 5 nights would be most worthwhile to properly resolve the nature of the subtle absorption profile changes. Finally, a reminder from Sect. 1, that photometric variability of up $\sim 0.02 \mathrm{mag}$ in $V$ has also been detected in HD 152408 (Balona 1982).

The weak emission features due to moderately ionized metals (e.g. N III $\lambda 4364$, C III $\lambda 5696$ ) are also approximately Gaussian in shape, with full-width at halfmaximum of $\sim 115 \mathrm{~km} \mathrm{~s}^{-1}$ (cf. $v_{\mathrm{e}} \sin (i) \sim 85 \mathrm{~km} \mathrm{~s}^{-1}$; Table 1) and centred close to rest velocity. Though they form in the expanding envelope of HD 152408, these features must nevertheless originate close to the stellar surface, but slightly beyond the CIV and He II absorption line-formation region. Previously, Prinja \& Fullerton (1994) provided tentative indication for a possible link between velocity shifts in these metal lines and the onset of propagating absorption enhancements in He I $\lambda 5876$. The radial velocities of the $\mathrm{N}$ III and $\mathrm{CIII}$ emission features were determined by Gaussian profile fitting, and the results are shown in Fig. 11 as a function of time. The central velocities are also compared in this figure to the stellar wind structures evident in He I $\lambda 5876$. There are genuine shifts in the emission line central velocities of up to $\sim 15 \mathrm{~km} \mathrm{~s}^{-1}$, but with no significant change in total equivalent width. There does not appear to be any

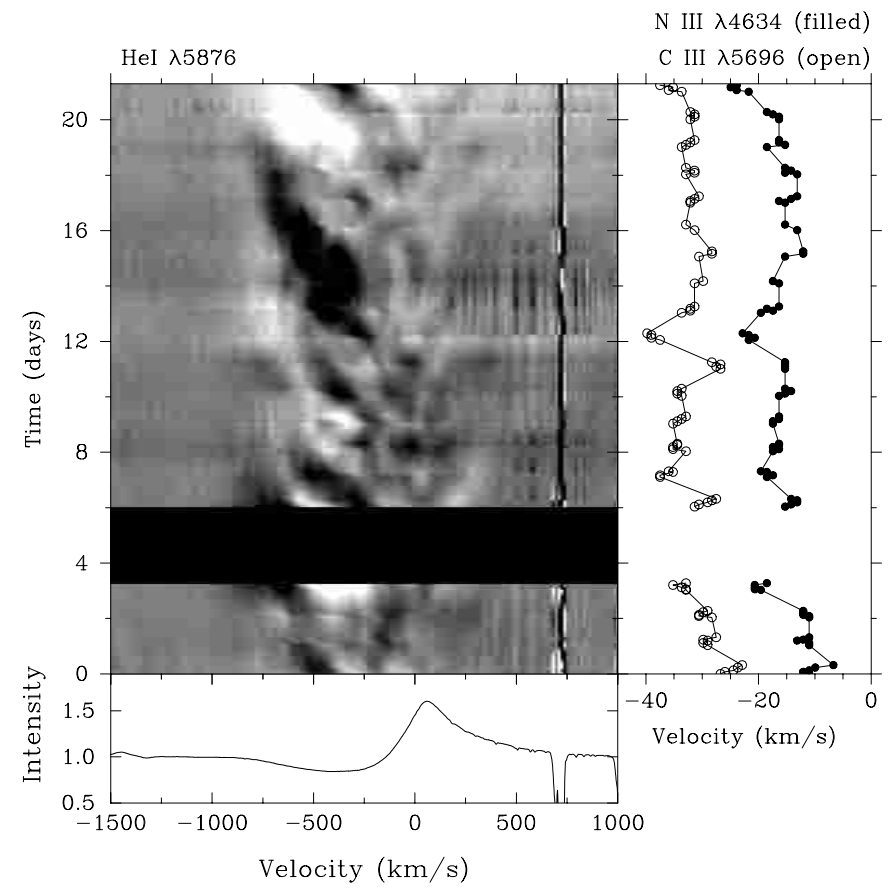

Fig. 11. (Heliocentric) radial velocity variations in deep-seated emission lines of $\mathrm{N}_{\text {III }} \lambda 4634$ and $\mathrm{C}_{\text {III }} \lambda 5696$ are shown as a function of time in the right-hand panel. These disturbances are compared to the onset and development of blue-ward migrating absorption features seen in He I $\lambda 5876$ (grey-scale; see also Fig. 3b). The mean FEROS He I $\lambda 5876$ profile is shown in the bottom-panel

unique relation between either the amplitude or direction of the velocity shifts and the occurrence of large-scale wind structure. Perhaps slightly intriguing is the relatively large disturbance in $\mathrm{N}$ III and $\mathrm{C}$ III central velocity seen between $T \sim 11$ to 12 days, which almost coincides with the initial development of one of the strongest absorption episodes in He I. Similar "associations" are not present, however, for velocity excursions seen at $T \sim 3$ days, 6 to 7 days, and 20 to 21 days. There is also no correlation between the peak intensity of the $\mathrm{N}$ III and $\mathrm{C}$ III emission and the central $\left( \pm 100 \mathrm{~km} \mathrm{~s}^{-1}\right) \mathrm{H} \alpha$ emission strength.

It is clear that there are variations in the radial velocities of weak metallic emission features which betray deep-seated near-photospheric perturbations. We cannot however recover in these emission-line data the 1.5 day period discovered above in the weaker $\mathrm{C}$ IV absorption lines. The emission fluctuations are not cyclic in our FEROS data set, though we cannot rule out the possibility of more subtle velocity variations in $\mathrm{N}$ III and $\mathrm{C}$ III on shorter timescales than considered here. These is also no significant power at $\sim 1.5$ days in He I and Balmer lines that exhibit discrete migrating features in the stellar wind, with a modulation time-scale of $\sim 7.7$ day (Sect. 4 ; Fig. 7 ).

\section{Long-term behaviour}

Our discussion has so far focussed on the more intensely time-sampled FEROS data secured between 1999 July to 


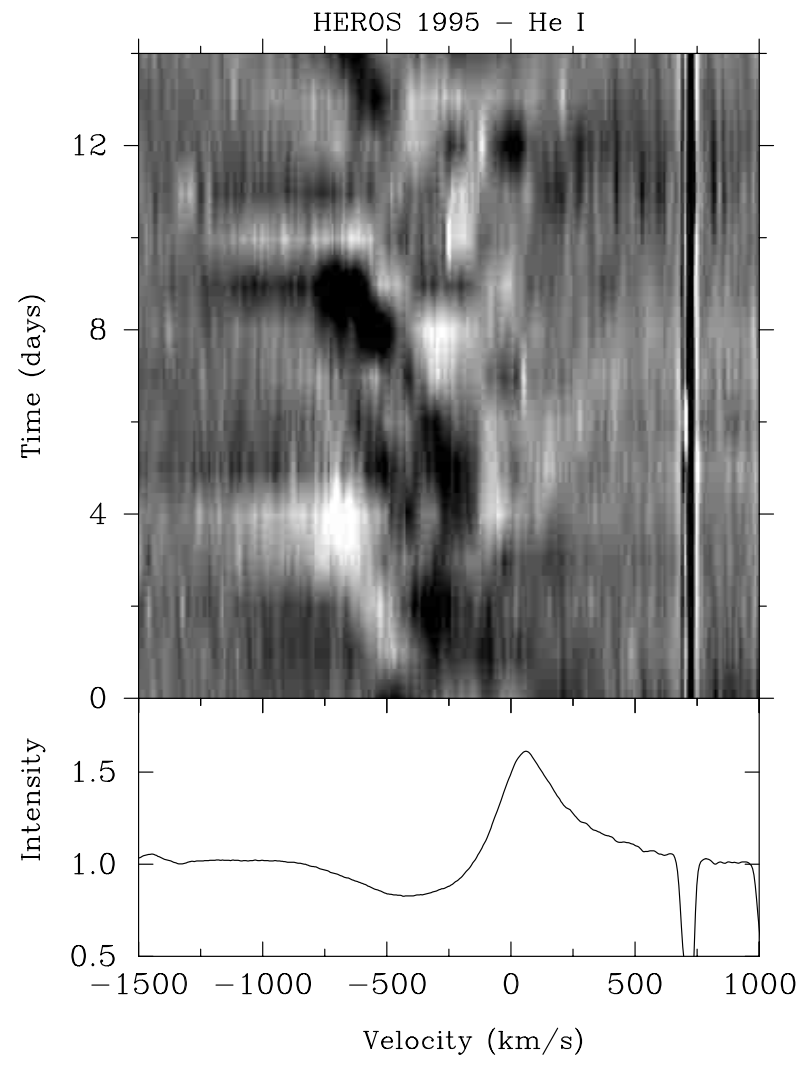

Fig. 12. Evidence for slowly evolving, blue-ward migrating, localised absorption features in He I $\lambda 5876$ spectra taken with the HEROS instrument in May-June 1995. Two features are identified between 3 to 9 days into the observing run, covering a velocity range of $\sim-200$ to $-750 \mathrm{~km} \mathrm{~s}^{-1}$

August, which is clearly very suitable for examining the incidence, development and recurrence of large-scale wind structure. As outlined in Sect. 2 (Table 2), we have secured other (more limited) observations of HD 152408 between 1995 and 1999 using the HEROS instrument. These additional data can provide some information on the longterm (year-to-year) properties of the stellar wind. Note also that several studies have previously reported optical line profile variability in this star (see references in Sect. 1), and in particular Prinja \& Fullerton (1994) documented blue-ward migrating discrete absorption features in the absorption trough of He I $\lambda 5876$, in data taken over $\sim 5$ nights during 1992 July. A grey-scale image of variability in He I $\lambda 5876$ in our HEROS data from 1995 (Table 2) is shown in Fig. 12 (cf. Fig. 3b). Fifteen spectra are presented covering $\sim 14$ days. Despite the poor time sampling we can identify at least two episodic features progressing from very low initial velocities. They accelerate very slowly towards the blue wing and are reminiscent of the features in the FEROS (1999) data. The data shown in Fig. 12 are of course not suitable for testing for quasi-periodic behaviour, or the $\sim 7.7$ and 3.9 day modulation times discussed in Sect. 4. (Unfortunately the same is true for all our HEROS data of HD 152408.) It is clear, however, that the basic observation of slowly migrating discrete features which affect a very wide velocity domain - both, blue- wards in absorption in $\mathrm{He}$ I and $\mathrm{H} \alpha$, and red-wards in the $\mathrm{H} \alpha$ emission wings - is a long-term (years) phenomena in this star. The underlying physical mechanism responsible for the variability is broadly stable over at least 8 years.

In addition to the episodic migrating features in the line profiles, we have shown that the $\mathrm{H} \alpha$ lines are also affected by changes in peak intensity and equivalent width, particularly in the low velocity regime. The central $\mathrm{H} \alpha$ equivalent width between $\pm 100 \mathrm{~km} \mathrm{~s}^{-1}$ was measured in the FEROS and HEROS 1995 data sets. The results are shown as a function of time in Fig. 13 (where time is normalised to the first exposure for each epoch). Gradual changes in peak $\mathrm{H} \alpha$ emission strength are better recorded in the more intensive FEROS data, though both data sets highlight continual fluctuations in the central regions. The overall mean equivalent width and standard deviation (s.d.) for this limited velocity region is $-7.5 \AA$, s.d. $0.4 \AA$ and $-7.4 \AA$, s.d. $0.3 \AA$ for FEROS and HEROS 1995, respectively. (The corresponding total equivalent widths across the full line profile are $-25.7 \AA$ and $-25.5 \AA$.) The data in Fig. 13 do not provide any reliable evidence for cyclic or periodic behaviour, including for example modulations which are closely commensurate with the periods identified in Sect. 4 for the (localised) propagating episodic features.

From the viewpoint of gross stellar wind properties, it is interesting to note that the time-averaged mean line profiles from epoch-to-epoch agree remarkably well. The mean $\mathrm{H} \alpha$ profile for each data set listed in Table 2 is shown in Fig. 14. The mean total $\mathrm{H} \alpha$ equivalent widths for the time-series data sets agree to within $\sim 0.8 \%$. This result ties in very well with the general success of deriving global parameters such as mass-loss rate from fitting $\mathrm{H} \alpha$ emission profiles in $\mathrm{OB}$ stars with steady-state line-driven-wind models (e.g. Leitherer 1988; Lamers \& Leitherer 1993; Puls et al. 1996). The temporal behaviour identified in our study does, however, indicate that the lower wind layers of HD 152408 (i.e. out to $\sim 3 R_{\star}$ ) are not smooth, but affected by pronounced coherent structures. Their influence is mostly restricted to localised and relatively shallow features on the broad overall $\mathrm{H} \alpha$ wings.

\section{Steady-state line-synthesis modelling}

In this section, we briefly attempt to match some of the $\mathrm{H} \alpha$ and He I line profiles of HD 152408 with steady-state line synthesis models. Our aim is simply to assess the extent of global mass flux changes that would be implied by the observations (though of course we do not suggest this as a physical interpretation of the variability). We also examine the plausibility of canonical wind velocity law parameterisations, and use our FEROS data to contrast the matches obtained from the line synthesis between $\mathrm{H} \alpha$ and He I $\lambda 5876$.

Crowther \& Bohannan (1997) and Bohannan \& Crowther (1999) have previously studied the timeaveraged optical and near-IR spectral properties of 


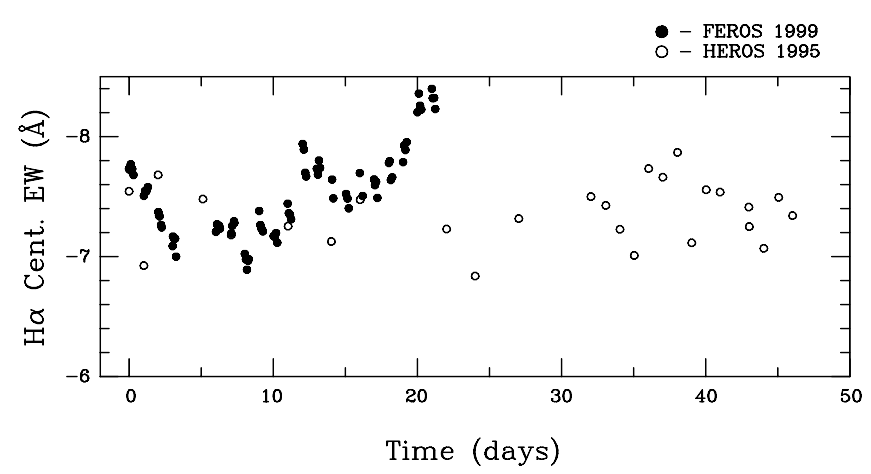

Fig. 13. Variations in the central $\left( \pm 100 \mathrm{~km} \mathrm{~s}^{-1}\right) \mathrm{H} \alpha$ emission equivalent width in FEROS (1999; closed symbols) and HEROS (1995; open symbols) data of HD 152408

HD 152408, revealing stellar parameters and chemistry with the non-LTE iterative technique of Hillier (1987, 1990). Here, we differ from these previous studies through application of the updated code of Hillier \& Miller (1998) which now accounts for line blanketing by $\mathrm{HI}$, He I-II, C III-IV, N III-V, Si IV, Fe IV-VI to generate synthetic line profiles for comparisons to our FEROS optical observations.

Use of a standard $\beta=1$ velocity law fails to produce the observed shape of the $\mathrm{H} \alpha$ profile (see Crowther \& Bohannan 1997). This is clearly illustrated in Fig. 15 where the mean FEROS $\mathrm{H} \alpha$ profile (open circles) is compared with two models, one calculated with a $\beta=1$ law (dashed line), the other using a tailored slower law (solid line), adopting $\beta 1=1, \beta 2=3, v_{\text {ext }}=500 \mathrm{~km} \mathrm{~s}^{-1}$ and $v_{\infty}=955 \mathrm{~km} \mathrm{~s}^{-1}$ using the parameterization of Hillier \& Miller (1999). Modelling the mean FEROS profiles of the usual He II $\lambda 4686$, He I $\lambda 5876$ and $\mathrm{H} \alpha$ diagnostics results in the following parameters for HD 152408 , i.e. $T_{*}=28 \mathrm{kK}$, $\log \left(L / L_{\odot}\right)=5.8, R_{\star}=34 R_{\odot}, \dot{M}=2.110^{-5} M_{\odot} \mathrm{yr}^{-1}$ and $\mathrm{H} / \mathrm{He}=1.5$ by number. Agreement with previous studies is excellent, revealing that the effects of line blanketing does not greatly affect the wind lines of such stars. Having fixed the stellar parameters from the above diagnostics, we subsequently calculated a range of models in which solely the mass-loss was varied, in order to mimic "extreme" FEROS cases.

Figure 15 also includes comparisons between the models and the mean He I $\lambda 5876$ profile. This reveals that the slow law also provides a better match to the emission component. However, both models strongly overestimate the absorption component. There is a well established problem with non-LTE models reproducing the absorption strengths of He I lines in Wolf-Rayet stars and O supergiants. Nevertheless, theoretical predictions for other optical lines are found to be in good agreement with observations, giving confidence in the reliability of derived parameters. From Fig. 15, absorption produced by the $\beta=1$ law, albeit too strong, is in better agreement with observation than the tailored slow law, since it extends much further to the blue.

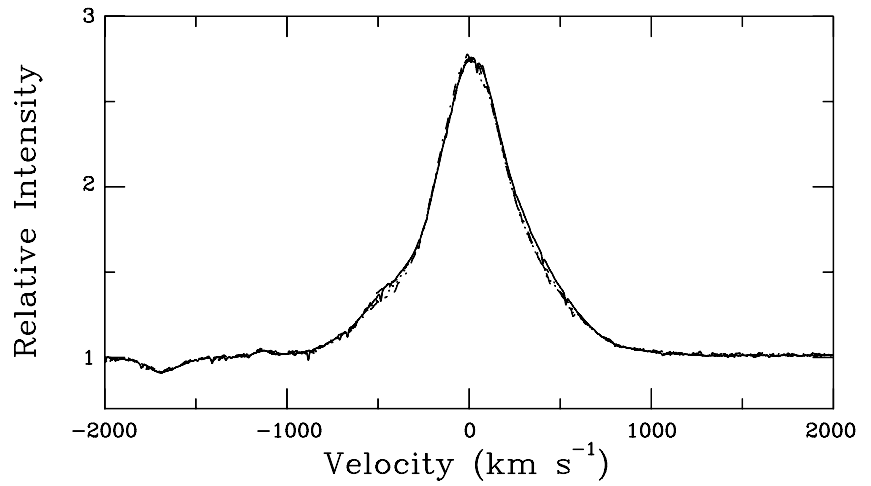

Fig. 14. Comparisons between the mean $\mathrm{H} \alpha$ profiles from FEROS (1999; solid line), HEROS 1995 (dotted line), HEROS 1996 (short-dashed line), and HEROS 1999 (dot-dashed line). The epoch-to-epoch mean line profiles are essentially identical, particularly in the central emission regions

We compare in Fig. 16 synthetic $\mathrm{H} \alpha$ and $\mathrm{He}$ I profiles with (constructed) FEROS minimum and maximum emission flux "template" profiles. The mass-loss required to reproduce the $\mathrm{H} \alpha$ profiles correspond to $2.010^{-5}$ and $2.410^{-5} M_{\odot} \mathrm{yr}^{-1}$. The observed $\mathrm{H} \alpha$ profile changes can be reproduced with a $\pm 10 \%$ variation in $\dot{M}$ - similar variations are predicted in He I $\lambda 5876$, in reasonable agreement for maximum emission, but somewhat discrepant for the minimum case. The He I absorption is more discrepant, however, since the models predict it to (i) be much deeper than observed, and (ii) extend to $\sim 75 \% v_{\infty}$, in contrast to $\sim v_{\infty}$ from observation.

\section{Discussion}

In this study, we have successfully probed time-dependent stellar wind activity in HD 152408 by simultaneously monitoring fluctuations in several strategic optical absorption and emission lines, including Balmer, He I, He II, and weak metallic features. The principal results on the nature of wind structure in HD 152408 may be summarised as follows:

1. The $\mathrm{H} \alpha$ forming (inner-stellar wind) region is dominated by very slowly evolving ( several days $)$ perturbations that are betrayed by localised absorption and emission features that propagate blue-ward and red-ward in Balmer and He I lines. These features are fairly pronounced, with changes of $\sim 10 \%$ of the line flux. They migrate over a wide velocity range, between $<0.05 v_{\infty}$ to $>0.9 v_{\infty}$;

2. There is a clear asymmetry between blue-ward and red-ward propagating features, such that the accelerations of the latter are barely $50 \%$ of the former. Variability in the red wing of $\mathrm{H} \alpha$ only extends to $~ 50 \%$ of the velocities compared to the blue wing;

3. At blue-ward velocities the perturbations in the stellar wind are seen as reduced flux (not enhanced emission) in the $\mathrm{H} \alpha$ wing, with excellent correspondence, in velocity and time, to the occurrence of discrete 

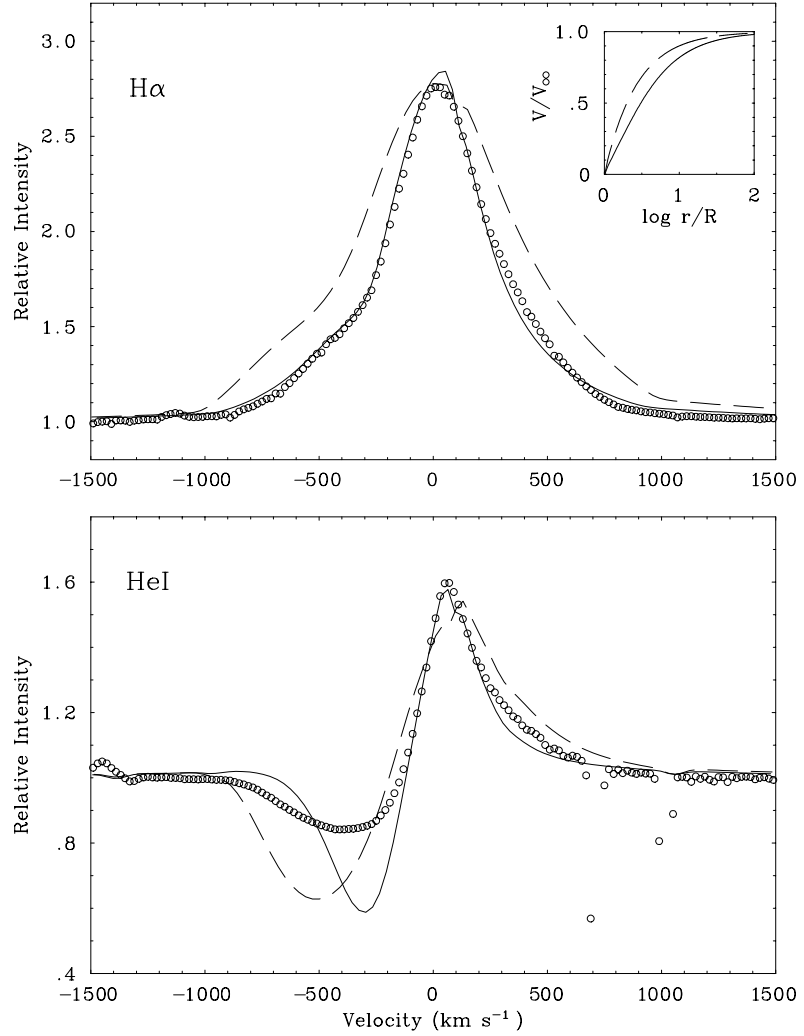

Fig. 15. Upper-panel - The mean FEROS $\mathrm{H} \alpha$ profile (open circles) is compared to synthesised model profiles, calculated for a $\beta=1$ velocity law (dashed line) and a (tailored) slower velocity law (solid line; see Sect. 7 ). The two velocity laws are compared in the inset. Lower-panel-same as above, except for He I $\lambda 5876$

absorption components in the $\mathrm{P}$ Cygni-like trough of He I $\lambda 5876$. The implication is that the $\mathrm{H} \alpha$ opacity in the wind of HD 152408 is extremely high and the source function permits line-scattering in a similar fashion to the formation mechanism operating in $\mathrm{He}$ I $\lambda 5876$. The blue-shifted perturbations can therefore be localised in the direction along the line-of-sight to the stellar disk;

4. The fundamental pattern of wind activity revealed by our time-series data is systematic and regular over a time-scale of several days, as opposed to chaotic over a few hours. (The latter may, for example, be expected for the case of small-scale structures arising from the action of the radiative instability mechanism.) Furthermore, we identify quasi-periodic modulation time-scales for changes in the He I and Balmer lines of $\sim 7.7$ and 3.9 days (the latter being the harmonic of the former). The extended length of these time-scales, compared to steady-state wind flow times across the optical line formation regions, suggests that the variability has a causal connection to stellar rotation;

5. The weak $\mathrm{C}$ IV $\lambda \lambda 5801,5812$ absorption lines are used to diagnose structure on or very close to the photosphere. Cyclic radial velocity shifts of up to $\sim 25 \mathrm{~km} \mathrm{~s}^{-1}$
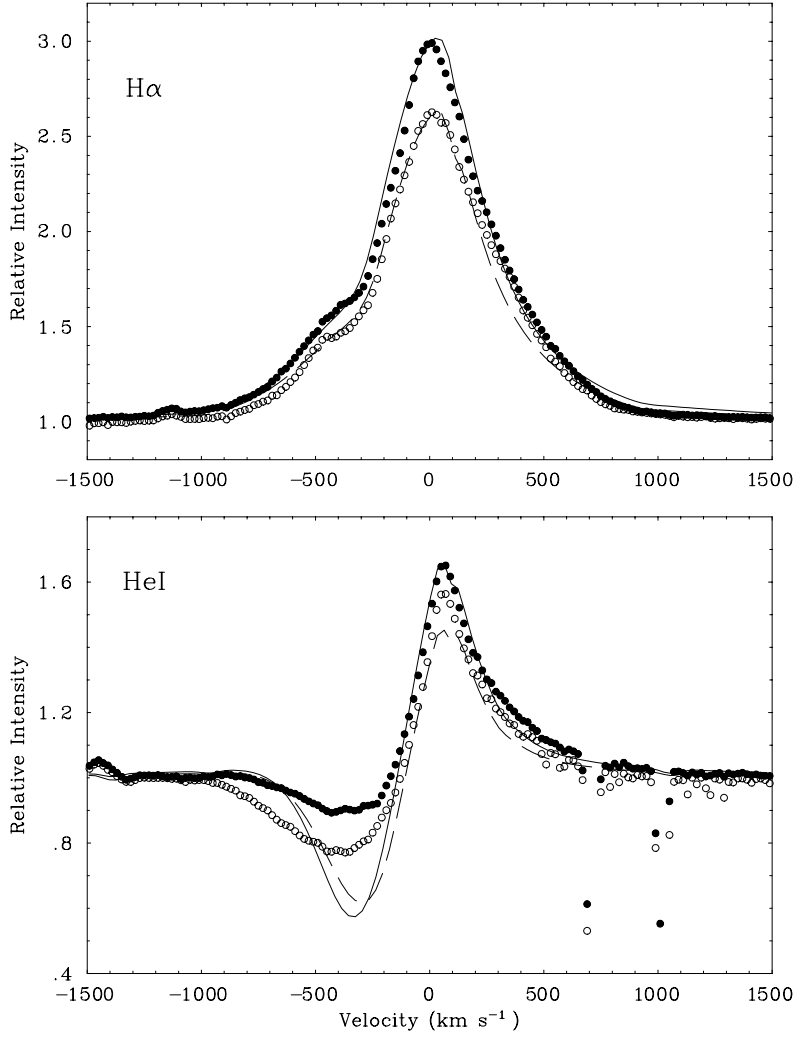

Fig. 16. Upper-panel - The FEROS maximum and minimum flux $\mathrm{H} \alpha$ profiles of HD 152408 (filled circles and open circles, respectively) are compared to synthetic model profiles for massloss rates of $2.410^{-5} M_{\odot} \mathrm{yr}^{-1}$ and $2.010^{-5} M_{\odot} \mathrm{yr}^{-1}$ (solid and dashed lines, respectively). Lower-panel - same as above, except for He I $\lambda 5876$

are detected in these lines, with a period of $\sim 1.5$ days (and possibly $\sim 2.3$ days);

6. The strong $\mathrm{H} \alpha$ emission line of HD 152408, in particular, reveals a second form of variability that is dominant in the central regions of the profile (i.e. $\pm 200 \mathrm{~km} \mathrm{~s}^{-1}$ ), and is characterised by significant changes in the peak emission flux over several days. There is no clear empirical connection evident between these peak fluctuations and the occurrence of discrete migrating features. In non-LTE line-synthesis modelling the differences in central emission may be reproduced by changes of $\pm 10 \%$ in the mass-loss rate.

\subsection{Co-rotating interaction regions?}

The key properties outlined above are broadly consistent with a scenario of perturbations in the stellar wind that create a plateau in the local radial velocity gradient, which in turn increases the line optical depth at that point in the flow. The spectroscopic signatures described here would therefore arise due to material that accelerates through the plateau much more rapidly that the plateau itself evolves. Since the absorption and emission features evolve over several days, this phenomenological picture is most closely related to the hydrodynamical models of 
co-rotating interaction regions (CIRs) developed by Cranmer \& Owocki (1996), which were inspired by the ideas discussed by Mullan (1984, 1986). The constraints offered to these models by our optical study are outlined below.

Some simple streak-lines are drawn in Fig. 17 to (phenomenologically) represent perturbations in the stellar wind, where the spiral shapes are solely due to conservation of angular momentum and acceleration in the wind due to " $\beta$-type" radial velocity laws. (We adopt the fundamental parameters in Table 1 and $\left.v_{\text {rot }}=v_{\mathrm{e}} \sin (i)\right)$. For reasonable radial wind velocity laws (i.e. $\beta \sim 1-2$; see e.g. Sect. 7), the streak-line present in the dominant $\mathrm{H} \alpha$ forming inner-wind region sampled in our observations $\left(\leq 3 \quad R_{\star}\right)$ is almost radial, with minimal azimuthal extent. Furthermore, in the $\beta=1$ case, the entire streakline within $3 R_{\star}$ appears in the line-of-sight and projected against the stellar disc for the $\sim 10 \%$ of the rotation period (in the equatorial plane). During this period the corresponding absorption feature would not be very localised in velocity space, but (unlike the observations) would extend simultaneously over $\sim 600 \mathrm{~km} \mathrm{~s}^{-1}$. The projected radial velocities in the central line-of-sight are also shown in Fig. 17 (lower-panel) for $\beta=1,2$, and 3 . The domain considered is again restricted to the inner-wind region $\left(\leq 3 R_{\star}\right)$. The longest-lived (blue-ward) discrete features evident in the He I line profiles of HD 152408 evolve over $\sim 8$ days, from $\leq 50 \mathrm{~km} \mathrm{~s}^{-1}$ to $\sim 900 \mathrm{~km} \mathrm{~s}^{-1}$ (see e.g. Fig. $3 \mathrm{~b}$ ). This progression cannot be reproduced with the "standard" velocity fields considered in these streak-lines, since in the $\beta=1$ case the acceleration is too rapid compared to the observations, and the slower $\beta$ laws (including the tailored law used in Sect. 7) do not extend to sufficiently high velocities within $3 R_{\star}$.

Probably the most severe constraint for a simple corotating stream picture comes from the fact that the accelerations of the red-ward migrating features are typically $\leq 50 \%$ of the values measured for blue-ward features (e.g. Figs. 3a and 5). This is rather difficult to reconcile, since a given red-ward perturbation is eventually expected to rotate into the line-of-sight in front of the stellar disc and yield the same velocity behaviour at blue-ward velocities. Even in the case of multiple rotating spiral structures, each with different (non-standard) velocity fields, discrete features would be anticipated with a range of accelerations, but with similar overall patterns in the blue-ward and redward portions of the line profiles. An option that may deserve attention in future hydrodynamical modelling is whether a CIR can form with different velocity fields in its leading (blue-ward; absorption) and trailing (red-ward; emission) edges. Schematically, we envisage two or more broad spiral structures in the wind, each with variable acceleration as a function of azimuth. Crudely, the $\beta=1$ and $\beta=2$ streak-lines in the Fig. 17 (upper-panel) may, for example, represent the leading and trailing edges of a single structure. A somewhat ad-hoc possibility here may be that the leading and trailing edges are optically thick, so that blue-ward absorption due to the trailing edge is not observed, and similarly red-ward emission due to the leading edge is not seen as the structure continues to rotate past the line-of-sight.

There are two other general empirical constraints provided by our results that are pertinent to the CIR model. Firstly, it is clear that the perturbations in the wind of HD 152408 are already well established and pronounced from very low velocities above the stellar surface, and throughout the inner-wind regions. Despite a modest stellar rotation rate, therefore, the "underlying" essentially radial wind material must meet the perturbation (CIR for example) almost immediately, in the deepest layers close to the photosphere. In the models of Cranmer \& Owocki (1996) enhanced absorption arises from an extended region of near-uniform velocity that marks the initial response of the unperturbed wind to the slower CIR ahead. The distance from the star of this plateau in the velocity gradient may be controlled by the amplitude of the perturbation, its azimuthal location on the surface, and the stellar rotation velocity.

Another point to note from our results of HD 152408 is that individual large-scale coherent wind structures survive over at least several days, and possibly much longer. These perturbations persist against a background of anticipated small-scale, stochastic wind structure that is predicted to arise from the action of radiative instabilities that are intrinsic to the outflow. The black troughs of saturated UV resonance line $\mathrm{P}$ Cygni profiles, soft $\mathrm{X}$-ray emission, and exceptionally small ratio of $v_{\infty} / v_{\text {edge }}$ (Sect. 1) in HD 152408, are all testiment to turbulent motions and randomly distributed shocks in the stellar wind. It is clear, however, that the small-scale structure does not substantially disrupt the large-scale (CIR-type) features in HD 152408. The preliminary simulations presented by Owocki (1999) suggest that in cases where unstable selfexcitation of the wind extends back to the base of the outflow, the stochastic small-scale structure can dominate and entirely destroy any large-scale features, including the extended velocity plateaus.

The origin of the CIRs relates to wind perturbation mechanisms that are rooted at the stellar surface. Velocity fields due to non-radial pulsation and a (weak) magnetic field configuration are clear contenders that have the potential to influence the emergence of a line-driven stellar outflow and thus trigger wind structure (see e.g. Henrichs et al. 1994; Cranmer \& Owocki 1996). We have demonstrated that systematic variability is present in absorption lines formed in the photospheric layers of HD 152408 (e.g. Fig. 10), but more details on the physical form of this surface structure must await a more intensive (short) time-series data set. It is also tempting to consider that the presence of a magnetic field in particular may provide significant variation in the mass-flux and thus also account for the fluctuations noted here in the central (peak) regions of the $\mathrm{H} \alpha$ emission line of $\mathrm{HD}$ 152408. If magnetic pressure plays a significant role in the near-star wind regions where the $\mathrm{H} \alpha$ line is formed, then perhaps as the star rotates a low-order field will present either regions 

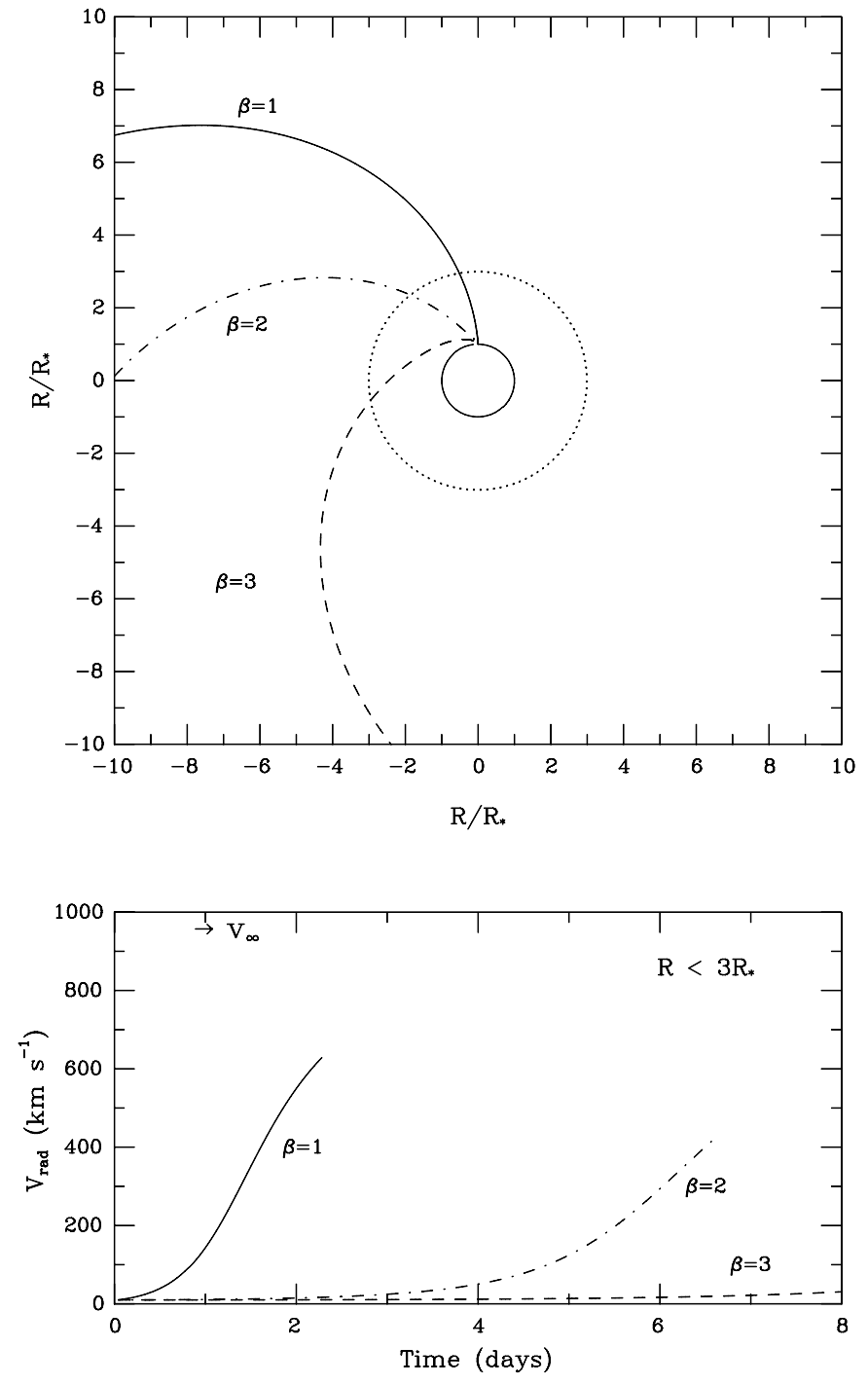

Fig. 17. Upper-panel: streak-lines are shown in the equatorial plane for $v_{\text {rot }}=85 \mathrm{~km} \mathrm{~s}^{-1}, v_{\infty}=955 \mathrm{~km} \mathrm{~s}^{-1}$, and $\beta$-type radial velocity laws; $\beta=1$ (solid line), $\beta=2$ (dot-dashed line) and $\beta=3$ (dashed line). The dominant $\mathrm{H} \alpha$-forming inner-wind region diagnosed by our optical spectroscopy of HD 152408 is approximately indicated by the dotted circle (i.e. less than $3 R_{\star}$ ). Lower-panel: the corresponding projected radial velocities as a function of time are shown for the three velocity laws considered above. In each case the velocities shown are restricted to a region $<3 R_{\star}$

where the flow is suppressed in "closed" (horizontal) field lines, or it is promoted in more "open" (vertical) lines. (Note, however, that we do not find evidence for periodic variations in the central $\mathrm{H} \alpha$ emission in our data.)

Following on from our study of time-variable structure in the active wind of HD 152408, we are now pursuing three avenues of research to extend and develop the results presented here; (i) sensitive searches for surface inhomogeneities and photospheric velocity fields in HD 152408, (ii) comparisons to large-scale wind structure evident in the optical wind lines of luminous Of stars, such as HD 151804, and (iii) exploiting millimetre and radio di- agnostics to constrain the geometric extent of large-scale structure in the outer winds, beyond the optical and UV line-formation regions.

Acknowledgements. We thank the staff at the European Southern Observatory for their hospitality. RKP is grateful to the British Council for travel support. We thank the DFG for support for the FEROS project at the Landessternwarte (Ap 19/6-1,6-2). We are also grateful to our collegues Ian Howarth, Tim Harries and Richard Townsend for helpful discussions.

\section{References}

Baade, D. 1988, in O. stars, \& Wolf-Rayet Stars, ed. P. S. Conti, A. B. Underhill, NASA SP-497, Washington, 137

Balona, L. A. 1982, MNRAS, 254, 404

Bohannan, B., \& Crowther, P. A. 1999, ApJ, 511, 374

Conti, P. S., Garmany, C. D., \& Hutchings, J. B. 1977, ApJ, 215,561

Cranmer, S. R., \& Owocki, S. P. 1996, ApJ, 462, 469

Crowther, P. A., \& Bohannan, B. 1997, ApJ, 317, 532

De Jong, J. A. 2000, Ph.D. Thesis, University of Amsterdam

Drew, J. E. 1985, MNRAS, 217, 867

Ebbets, D. 1982, ApJS, 48, 399

Eversberg, T., Lépine, S., \& Moffat, A. F. J. 1998, ApJ, 494, 799

Fullerton, A. W. 1990, Ph.D. Thesis, University of Toronto

Fullerton, A. W., Massa, D. L., Prinja, R. K., Owocki, S. P., \& Cranmer, S. R. 1997, A\&A, 327699

Fullerton, A. W., Gies, D. R., \& Bolton, C. T. 1996, ApJ, 390, 650

Gies, D. R., \& Kullavanijaya, A. 1988, ApJ, 326, 813

Glatzel, W. 1999, in Variable and Non-spherical Stellar Winds in Luminous Hot Stars, ed. B. Wolf, O. Stahl, \& A. W. Fullerton (Springer, Heidelberg), 345

Henrichs, H. F., Kaper, L., \& Nicols, J. S. 1994, in Rotation, Pulsation, and Mass-loss in Early-type Stars, ed. L. A. Balona, H. F. Henrichs, \& J. M. Le Contel (Kluwer, Dordrecht), 517

Hillier, D. J. 1987, ApJS, 63, 947

Hillier, D. J. 1990, A\&A, 231, 111

Hillier, D. J., \& Miller, D. L. 1998, ApJ, 496, 407

Hillier, D. J., \& Miller, D. L. 1999, ApJ, 519, 354

Howarth, I. D., Prinja, R. K., \& Massa, D. 1995, ApJ, 452, L65

Howarth, I. D., Seibert, K. W., Hussain, A. J., \& Prinja, R. K. 1997, MNRAS, 284, 265

Kaper, L., Henrichs, H. F., Fullerton, A. W., et al. 1997, A\&A, 327,281

Kaper, L., Fullerton, A. W., Baade, D., et al. 1998, in Cyclical Variability in Stellar Winds, ed. L. Kaper, \& A. W. Fullerton (Springer, Heidelberg), 103

Kaper, L., Henrichs, H. F., Nichols, J. S., et al. 1996, A\&AS, 116257

Kaufer, A., Stahl, O., Tubbesing, S., et al. 2000, SPIE proceedings, vol. 4008, Munich, Germany

Kiriakidis, M., Fricke, K. J., \& Glatzel, W. 1993, MNRAS, 264, 50

Lamers, H. J. G. L. M., \& Leitherer, C. 1993, ApJ, 412, 771

Leitherer, C. 1988, ApJ, 326, 356

Lovy, D., Maeder, A., Noëls, A., \& Gabriel 1984, A\&A, 133, 307

Massa, D., et al. 1995, ApJ, 452, L53 
Moffat, A. F. J., \& Robert, C. 1992, in Nonisotropic and Variable Outflows from Stars, ed. L. Drissen, C. Leitherer, \& A. Nota (ASP, San Francisco), 203

Morrison, N. D. 1975, ApJ, 200, 113

Mullan, D. J. 1984, ApJ, 283, 303

Mullan, D. J. 1986, A\&A, 165, 157

Owocki, S. P. 1999, in Variable and Non-spherical Stellar Winds in Luminous Hot Stars, ed. B. Wolf, O. Stahl, \& A. W. Fullerton (Springer, Heidelberg), 294

Prinja, R. K., Massa, D., Howarth, I. D., \& Fullerton, A. W. 1998, MNRAS, 301, 926
Prinja, R. K., \& Fullerton, A. W. 1994, ApJ, 426, 345

Prinja, R. K., Barlow, M. J., \& Howarth, I. D. 1990, ApJ, 361 607

Puls, J., Kudritzki, R. P., Herrero, A., Pauldrach, A. W. A., \& Haser S. M. 1996, A\&A 305, 171

Roberts, D. H., Lehár, J., \& Dreher, J. W. 1987, AJ, 93968

Struve, O. 1944, ApJ, 100, 189

Swings, P. 1942, ApJ, 95, 112

Walborn, N. R. 1972, AJ, 77, 312

Walborn, N. R. 1975, PASP, 87, 735

Walborn, N. R., \& Fitzpartick, E. L. 2000, PASP, 112, 50 